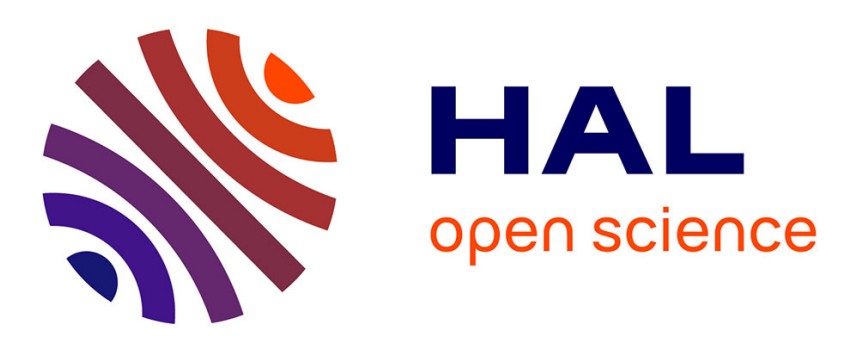

\title{
Asymptotic wavelet and Gabor analysis: extraction of instantaneous frequencies
}

\author{
Nathalie Delprat, Bernard Escudié, Philippe Guillemain, Richard \\ Kronland-Martinet, Philippe Tchamitchian, Bruno Torresani
}

\section{To cite this version:}

Nathalie Delprat, Bernard Escudié, Philippe Guillemain, Richard Kronland-Martinet, Philippe Tchamitchian, et al.. Asymptotic wavelet and Gabor analysis: extraction of instantaneous frequencies. IEEE Transactions on Information Theory, 1992, 10.1109/18.119728 . hal-01222729

\section{HAL Id: hal-01222729 \\ https://hal.science/hal-01222729}

Submitted on 30 Oct 2015

HAL is a multi-disciplinary open access archive for the deposit and dissemination of scientific research documents, whether they are published or not. The documents may come from teaching and research institutions in France or abroad, or from public or private research centers.
L'archive ouverte pluridisciplinaire HAL, est destinée au dépôt et à la diffusion de documents scientifiques de niveau recherche, publiés ou non, émanant des établissements d'enseignement et de recherche français ou étrangers, des laboratoires publics ou privés. 


\title{
ASYMPTOTIC WAVELET AND GABOR ANALYSIS: EXTRACTION OF INSTANTANEOUS FREQUENCIES ${ }^{\$}$
}

\author{
N. Delprat ${ }^{(1)}$, B. Escudié (2), P. Guillemain (1) \\ R. Kronland-Martinet ${ }^{(1)(3)}, \mathrm{Ph}$. Tchamitchian ${ }^{(4)}, \mathrm{B}$. Torrésani \\ Centre de Physique Théorique \\ CNRS-Luminy Case 907 F-13288 Marseille Cedex 09 \\ FRANCE
}

\begin{abstract}
Dedicated to Alex GROSSMANN, who taught us the importance of the phase
\end{abstract}

\begin{abstract}
:
We investigate the behaviour of the continuous wavelet and Gabor coefficients in the asymptotic limit, using stationary phase approximations. In particular, we show how, under some additional assumptions, these coefficients allow the extraction of some characteristics of the analysed signal, like for example frequency and amplitude modulation laws. We also briefly discuss applications to spectral lines estimations and matched filtering.
\end{abstract}

\section{I: INTRODUCTION:}

Time-frequency representations, as for instance spectrograms, scalograms, or bilinear representations like Wigner or smoothed Wigner distributions are now commonly used in signal analysis to investigate the time-frequency content of an analysed signal. Most of the algorithms that have been developed in that context are essentially based on the study of the "time-frequency energy localisation", the representation or its squared-modulus in the linear case being interpreted as an energy density in the time-frequency plane. Among the linear representations, the Gabor representation [Ga] (or sliding window Fourier transform), based on time and frequency translations, has been the most popular for a long time. More recently, Grossmann and Morlet [Gr-Mo] proposed an alternative representa-

\$ Work partially supported by the GDR Ondelettes of CNRS

(1) LMA-IM, 31 Chemin J. Aiguier, F-13009 Marseille, France

(2) LTS-ICPI Lyon, 25 rue du Plat, F-69288 Lyon, Cedex 02, France

(3) Currently at CCRMA, Stanford University

(4) Also at Faculté des sciences et techniques de Saint-Jérome, Marseille 
tion, called the wavelet transform, which basically has the same structure [Gr-Mo-Pa1], the frequency translations being replaced by dilations (the dilation parameter being interpreted as the quotient of a reference frequency by a frequency shift). Both methods have been applied to signal analysis, with comparable performance levels, in different contexts.

The mathematical theory of continuous wavelet analysis and Gabor analysis, and their discretizations (frames), has been mainly developed in [Da1]. In particular, it has been shown that there exists an important difference between these two representations, at the level of discretization. Indeed, while it is convenient to sample uniformly the time and frequency parameters in the Gabor case, the time and scale parameters have to be sampled on a dyadic lattice in the wavelet case. This is actually the consequence of a strategic difference: Gabor functions perform an analysis with constant absolute bandwidth $\Delta \nu$, while wavelets perform an analysis with constant relative bandwidth $\frac{\Delta \nu}{\nu}$.

We consider here their application to specific problems of signal analysis, namely the problems of extraction and characterisation of signals modulated in amplitude and frequency, in the asymptotic (i.e. high frequency) limit. More specificaly, we are interested not only in the asymptotic signals themselves, for which standard techniques based on Hilbert transform in general give good results, but rather on composite signals of asymptotic type, which can be defined as sums of asymptotic signals. Note that a composite signal of asymptotic type has a priori no reason to be itself asymptotic; moreover, the Hilbert transform is in general unable to give precise informations about the characteristics of the components separately, like for instance frequency and amplitude modulation laws. Nevertheless, the first tests of the algorithms presented here will be performed on asymptotic signals.

The plan of the paper is as follows: After briefly recalling in section II the basic definitions and properties of wavelet and Gabor analysis, and standard results on frequency and amplitude modulated and asymptotic signals in section III, we describe in section IV the behaviour of wavelet and Gabor analysis in the asymptotic limit, for asymptotic analysing functions. We show in section $\mathrm{V}$ how, for Gaussian analysing functions, the asymptotic assumption on the analysing function can be relaxed. Algorithms are proposed, which allow the extraction of instantaneous frequencies and spectral lines, and in some cases the separation of frequency-modulated components is a signal. Numerical results are presented in section VI, showing in particular that the study of the phases of wavelet and Gabor coefficients allow the construction of algorithms much more precise than those based on the energy localisation criteria. Applications to matched filtering are discussed in section VII, and section VIII is devoted to conclusions. 


\section{II: CONTINUOUS WAVELET AND GABOR ANALYSIS:}

We describe in this section the basic properties of continuous wavelet and Gabor analysis, in the form we will use in this paper. For more details on such techniques, we refer to [Da1], [Gr-Mo], [Gr-Mo-Pa1], [Gr-Mo-Pa2] and [Gr-KM-Mo].

\section{II-1: Wavelet analysis:}

We will restrict here to the wavelet analysis of the real Hardy space:

$$
H^{2}(\mathbf{R})=\left\{f \in L^{2}(\mathbf{R}) ; \hat{f}(\omega)=0 \text { for } \omega \leq 0\right\}
$$

which is sufficient for our purpose. The analysis of $L^{2}(\mathbf{R})$ follows from simple modifications.

Start from two functions $g, h \in H^{2}(\mathbf{R})$, such that the following admissibility condition holds:

$$
0<\left|c_{g, h}\right|=\left|\int_{0}^{+\infty} \frac{\overline{\hat{g}(\omega)} \hat{h}(\omega)}{\omega} d \omega\right|<\infty \quad(I I-1)
$$

where our convention for Fourier transform is the following:

$$
\hat{f}(\omega)=<f, \varepsilon_{\omega}>_{L^{2}}=\int_{-\infty}^{+\infty} f(t) e^{-i \omega t} d t
$$

with $\varepsilon_{\lambda}(t)=e^{i \lambda t} . g$ (resp. $h$ ) is the analysing wavelet (resp. the reconstructing wavelet). To these functions are associated the wavelets, i.e. the functions $g_{(b, a)}$ and $h_{(b, a)}$, defined by:

$$
g_{(b, a)}(t)=\frac{1}{a} g\left(\frac{t-b}{a}\right)
$$

(with the same definition for $h_{(b, a)}$ ), where the translation and dilation parameters run over the Poincaré half plane: $\mathbf{H}=\left\{(b, a) \in \mathbf{R}^{2} ; a>0\right\}$ (notice that we have chosen here a different normalisation for the wavelets than that of [Gr-Mo]). The admissibility condition then implies the following resolution of the identity: to each $s \in H^{2}(\mathbf{R})$ one can associate the following family of coefficients (wavelet coefficients):

$$
T_{s}(b, a)=<s, g_{(b, a)}>_{L^{2}}
$$

which are simply the $L^{2}$ products of $s(t)$ by the dilated and translated wavelets (when there is no possible confusion, the subscript $s$ of $T_{s}$ will be omitted). One then has a reconstruction formula:

$$
s=\frac{1}{c_{g, h}} \int_{0}^{+\infty} \int_{-\infty}^{+\infty} T_{s}(b, a) h_{(b, a)} \frac{d a d b}{a}
$$


which expresses $s(t)$ as a sum of dilated and translated wavelets $h_{(b, a)}$, the coefficients of the decomposition being the corresponding wavelet coefficients (the equality being understood in the $L^{2}$ sense). In other words, the wavelets are used exactly as if they formed a biorthogonal basis, although this is far from being the case, the information provided by $T_{s}$ being highly redundant.

It may also be convenient in practice to use a slightly modified version of wavelet analysis, which is simply to analyse and reconstruct a $\lambda$-frequency-shifted version of $s(t)$ instead of $s$ itself, for some real number $\lambda$. One then has a the following decomposition of $s(t)$ :

$$
s=\frac{1}{c_{g, h}} \int_{0}^{+\infty} \int_{-\infty}^{+\infty} T_{s}^{\lambda}(b, a) h_{(b, a)} \varepsilon_{\lambda} \frac{d a d b}{a}
$$

which expresses $\varepsilon_{-\lambda} s(t)$ as a sum of dilated and translated wavelets $h_{(b, a)}$, the coefficients of the decomposition being the corresponding modified wavelet coefficients.

$$
T_{s}^{\lambda}(b, a)=<s, \varepsilon_{\lambda} g_{(b, a)}>_{L^{2}}
$$

For simplicity, we will write $T_{s}=T_{s}^{0}$.

It is often convenient to work with wavelets having good localisation properties in both the direct space and the Fourier space; in general $g$ can be chosen so that both $g$ and $\hat{g}$ have exponential decay at infinity (and/or the same for $h$ and $\hat{h}$ ). This moreover allows to interpret the dilation parameter $a$ as the inverse of a frequency shift parameter (up to a multiplicative constant, characteristic of the analysing wavelet), and then to make the connexion with Gabor coefficients.

Note that it is very useful for numerical applications to use a simplified version of the reconstruction formula:

$$
s(t)=\frac{1}{k_{g}} \int_{0}^{+\infty} T_{s}^{\lambda}(t, a) \varepsilon_{\lambda}(t) \frac{d a}{a}
$$

which expresses $s(t)$ as a sum over frequencies, with logarithmic measure. This is equivalent to take formally $h=\delta$ in eq. $(I I-5)$, the admissibility condition reading:

$$
0<\left|k_{g}\right|=\left|\int_{0}^{+\infty} \frac{\overline{\hat{g}(\omega)}}{\omega} d \omega\right|<\infty
$$

Assuming that $0<c_{g}:=c_{g, g}<\infty$, one then has that $T_{s}^{\lambda} \in L^{2}\left(\mathbf{H}, \frac{d a d b}{a}\right)$. However, the wavelet coefficients do not span the whole $L^{2}\left(\mathbf{H}, \frac{d a d b}{a}\right)$, but rather a subspace of it, called a reproducing kernel space:

$$
\mathcal{H}_{g}=\left\{F \in L^{2}\left(\mathbf{H}, \frac{d a d b}{a}\right) ; K_{g, h} . F=F\right\}
$$

with reproducing kernel given by:

$$
K_{g, h}\left(b, a ; b^{\prime}, a^{\prime}\right)=\frac{1}{c_{g, h}}<h_{\left(b^{\prime}, a^{\prime}\right)}, g_{(b, a)}>
$$


with respect to the measure $\frac{d a d b}{a}$ on the Poincaré half plane. It is worth noticing that $K_{g, h}$ is a projection operator, which is an orthogonal projector if and only if $h=g$. Notice that while the kernel depends on both $g$ and $h$, the reproducing kernel space only depends on the analysing wavelet $g$. The reproducing kernel property can in particular be used to recover wavelet coefficients by interpolation from a discretised version of the wavelet transform.

To understand the information provided by the wavelet coefficients, a standard technique [Gr-KM-Mo] is to code them into two images, representing respectively $\left|T_{s}^{\lambda}(b, a)\right|$ and $\Psi^{\lambda}(b, a)=\operatorname{Arg}\left[T_{s}^{\lambda}(b, a)\right]$. Still assuming that $0<c_{g}:=c_{g, g}<\infty$, thanks to the Plancherel equality:

$$
\|s\|^{2}=\frac{1}{c_{g, g}} \int\left|T_{s}^{\lambda}(b, a)\right|^{2} \frac{d a d b}{a}
$$

$\left|T_{s}^{\lambda}(b, a)\right|^{2}$ is interpreted as an energy density in the Poincaré half plane, and is often refered to as scalogram. We refer to [Gr-KM-Mo] for a description of the usual conventions and interpretations of the images. We will mainly focus here on the study of the phase $\Psi^{\lambda}(b, a)$ of the wavelet transform $T^{\lambda}$. 


\section{II-2: Gabor analysis:}

Let us describe now the Gabor analysis of $L^{2}(\mathbf{R})$ (and not $H^{2}(\mathbf{R})$ as in the wavelet case, since $H^{2}(\mathbf{R})$ is not stable by frequency translations), in a similar way than wavelet analysis (note that Gabor [Ga] was only concerned with Gaussian analysing and reconstructing functions; we use the term Gabor analysis for simplicity). Let $g, h \in L^{2}(\mathbf{R})$, and consider the time and frequency shifted copies of them, denoted by:

$$
g_{(b, \omega)}(t)=e^{i \omega(t-b)} g(t-b)
$$

and the same notation for $h_{(b, \omega)}$. One then has the following resolution of the identity: To each $s \in L^{2}(\mathbf{R})$, one associates the corresponding Gabor coefficients:

$$
G_{s}(b, \omega)=<s, g_{(b, \omega)}>_{L^{2}}
$$

from which one has the reconstruction formula, the integral converging strongly:

$$
s=\frac{1}{2 \pi<h, g>} \int_{-\infty}^{+\infty} \int_{-\infty}^{+\infty} G(b, \omega) h_{(b, \omega)} d \omega d b
$$

which expresses $s(t)$ as a decomposition over time and frequency shifted copies of a basic function. $g(t)$ (resp. $h(t)$ ) is called the analysing (resp. reconstructing) Gabor function, and the $g_{(b, \omega)}$ and $h_{(b, \omega)}$ the Gabor functions. Note that contrary to the case of the dilation parameter of wavelets, the frequency shift parameter of Gabor functions run over the whole real axis.

As in the previous section, the Gabor coefficients do not span the whole $L^{2}\left(\mathbf{R}^{2}, d b d \omega\right)$, but rather some reproducing kernel subspace of it, also denoted by $\mathcal{H}_{g}$ and defined by eq. $(I I-9)$, the reproducing kernel being now given by:

$$
K_{g, h}\left(b, \omega ; b^{\prime}, \omega^{\prime}\right)=\frac{1}{2 \pi<h, g>}<h_{\left(b^{\prime}, \omega^{\prime}\right)}, g_{(b, \omega)}>
$$

and still defining a projection operator, orthonormal if and only if $h=g$.

One usually represents the Gabor coefficients by two images, namely the modulus and the argument of the coefficients. As in the wavelet case, a Plancherel equality allows the interpretation of $|G(b, \omega)|^{2}$ as an energy density, usually called spectrogram, in the time-frequency plane. The argument of $G(b, \omega)$ represents a complementary information that we will study here. 


\section{III: ASYMPTOTIC SIGNAL AND EXPONENTIAL MODEL:}

Throughout sections III, IV and V, we will describe estimations techniques for wavelet and Gabor coefficients in the asymptotic limit. More specifically, we will derive asymptotic developments for such coefficients. As a convention, we will use the symbol $x \approx y$ to specify that the expression $x$ is approximated by the first order term $y$ in its asymptotic development.

Let us first briefly describe here the basic definitions and properties of asymptotic signals.

\section{III-1: The Canonical Representation of a Real Signal:}

An arbitrary real signal $s(t)$ can always be represented in terms of instantaneous modulus and argument, in the form:

$$
s(t)=A(t) \cos (\phi(t))
$$

Obviously, such a representation is far from being unique, in the sense that to a given real signal $s(t)$ one can associate an infinity of pairs $(A, \phi)$ such that eq. $(I I I-1)$ holds. Several authors (see e.g. [Pi-Ma]) have noticed that among these pairs, it is convenient to specify a particular one, called the canonical pair, defined as follows: consider the Hilbert transform $\mathcal{H}$, defined by:

$$
\mathcal{H}=-i \mathcal{F}^{-1} . \varepsilon \cdot \mathcal{F}
$$

where $\mathcal{F}$ is the Fourier transform, and $\varepsilon$ is defined by its action on functions, according to:

$$
(\varepsilon . f)(\omega)=\operatorname{Sgn}(\omega) f(\omega)
$$

For instance, if $\alpha \in \mathbf{R}$, one obviously has that:

$$
[\mathcal{H} \cdot \cos ](\alpha t)=\sin (\alpha t)
$$

By definition, the analytic signal $Z_{s}(t)$ associated with $s(t)$ is obtained by a linear filtering of $s(t)$ cancelling its negative frequencies, which can be expressed as:

$$
Z_{s}(t)=[\mathbf{1}+i \mathcal{H}](t)
$$

$Z_{s}$ is completely characterised by the pair $\left(A_{s}, \phi_{s}\right)$ by setting:

$$
Z_{s}(t)=A_{s}(t) \exp \left(i \phi_{s}(t)\right)
$$

if one assumes that $A_{s}$ is non-negative, and that $\phi_{s}$ takes its values in the interval $[0,2 \pi[$. $\left(A_{s}, \phi_{s}\right)$ is called the canonical pair associated to $s(t)$. Obviously,

$$
\operatorname{Re}\left(Z_{s}\right)=s
$$


can be written as:

$$
s(t)=A_{s}(t) \cos \left(\phi_{s}(t)\right)
$$

which defines its canonical representation.

The canonical representation of $s(t)$ allows the introduction of the instantaneous frequency $\nu_{s}(t)$ of $s(t)$, defined by:

$$
\nu_{s}(t)=\frac{1}{2 \pi} \frac{d \phi_{s}}{d t}
$$

Note that while the definition of the instantaneous frequency always makes sense mathematically, its physical significance can be doubtful in some particular situations, for instance when $s(t)$ is not oscillating enough, i.e. if $\phi_{s}$ varies slowly compared to $A_{s}$, or when $s(t)$ is the sum of two sine functions.

Several authors have studied the problem of characterizing the possible canonical pairs $\left(A_{s}, \phi_{s}\right)$, in general by using spectral methods. Some sufficient conditions are derived in [Pi-Ma].

\section{III-2: Asymptotic Analytic Signal, and Exponential Model:}

Let $s \in L^{2}(\mathbf{R})$ be a real finite energy signal, of the form $(I I I-1)$, where $A(t) \geq 0$ and $\phi(t) \in[0,2 \pi[$ for all $t \in \mathbf{R}$. Assume moreover that $s(t)$ is asymptotic, which essentially means that (see [Co], [Di]):

$$
\left|\frac{d \phi}{d t}\right| \gg\left|\frac{1}{A} \frac{d A}{d t}\right|
$$

and that the signal $s(t)$ is locally monochromatic. An explicit form for the associated analytic signal can be obtained by using the stationary phase method (see appendix). Set:

$$
s_{ \pm}(t)=\frac{1}{2} A(t) e^{ \pm i \phi(t)}
$$

and evaluate the corresponding Fourier transforms $\widehat{s}_{ \pm}(\omega)$. Denoting by $t_{ \pm}(\omega)$ the corresponding stationary points of $\phi$, defined by:

$$
\phi^{\prime}\left(t_{ \pm}\right)= \pm \omega
$$

then:

$$
\widehat{s}_{ \pm}(\omega) \approx \sqrt{\frac{\pi}{2}} \frac{A\left(t_{ \pm}\right) e^{ \pm i \phi\left(t_{ \pm}\right)}}{\sqrt{\left|\phi^{\prime \prime}\left(t_{ \pm}\right)\right|}} e^{-i \omega t_{ \pm}} e^{i \delta_{ \pm}}
$$

where :

$$
\delta_{ \pm}=\frac{\pi}{4} S g n\left[\phi^{\prime \prime}\left(t_{ \pm}\right)\right]
$$

Consider the analytic signals:

$$
\widehat{Z}_{ \pm}(\omega)=2 H(\omega) \widehat{s}_{ \pm}(\omega)
$$


$H$ being the Heaviside step function. Let $Z(t)=Z_{+}(t)+Z_{-}(t)$, and:

$$
\begin{gathered}
Z_{+}(t)=\frac{1}{2 \pi} \int \widehat{Z}_{+}(\omega) e^{i \omega t} d \omega \\
=\frac{1}{\sqrt{2 \pi}} \int_{0}^{\infty} \frac{A\left(t_{+}\right) e^{i\left[\phi\left(t_{+}\right)+\omega\left(t-t_{+}\right)+\delta_{+}\right]}}{\sqrt{\left|\phi^{\prime \prime}\left(t_{+}\right)\right|}} d \omega
\end{gathered}
$$

Using again the stationary phase method to evaluate the integral, the essential contribution to $Z_{+}(t)$ is given by the stationary points $\omega_{+}$of the argument of the integrand, such that:

$$
\frac{\partial t_{+}}{\partial \omega}\left[\phi^{\prime}\left(t_{+}\right)-\omega_{+}\right]+t-t_{+}=0
$$

i.e. the points $\omega \in \mathbf{R}_{+}$such that:

$$
t_{+}(\omega)=t
$$

The set of such points is non-empty only if $\phi^{\prime}(t)>0$. Since moreover:

$$
\left[\frac{\partial t_{+}}{\partial \omega}\right] \phi^{\prime \prime}\left(t_{+}\right)=1
$$

eq. $(I I I-16)$ yields:

$$
Z_{+}(t)=H\left[\phi^{\prime}(t)\right] A(t) e^{i \phi(t)}
$$

The same argument gives:

$$
Z_{-}(t)=H\left[-\phi^{\prime}(t)\right] A(t) e^{i \phi(t)}
$$

which shows that:

$$
Z(t) \approx A(t) e^{i \phi(t)}
$$

In the asymptotic limit, the analytic signal of $A(t) \cos (\phi(t))$ is then close to the exponential model $A(t) \exp (i \phi(t))$. This property will be used in the following, in particular for numerical applications, where computations will be performed on real signals. 


\section{IV: THE CASE OF ASYMPTOTIC ANALYSING FUNCTION:}

We now turn to the description of the wavelet (resp. Gabor) coefficients in the asymptotic limit. Assuming that the signal and the wavelets (resp. Gabor functions) are all asymptotic allows to get an approximate expression for the corresponding coefficients, by means of the evaluation of the scalar products by the stationary phase method. As we will see, this allows the specification of some particular sets of curves in the time-scale halfplane (resp. the time-frequency plane), namely the ridge and the wavelet curves (resp. the Gabor curves). The ridge has an interesting property: it describes the frequency modulation law of the signal.

One is then led to a simple geometrical description of the transform (the so-called factorisation property): essentially, the restriction of the transform to the ridge (the socalled skeleton) reproduces the analytic signal of the analyzed signal, while the restriction to a given wavelet curve (resp. Gabor curve) reproduces the corresponding wavelets (resp. Gabor functions).

We first describe here the computation of the wavelet (resp. Gabor) coefficients in the asymptotic limit, and introduce the notions of ridge and wavelet (resp. Gabor) curves; we then derive simple formulas, allowing to build algorithms for the extraction of the ridge.

\section{IV-1: Wavelet Analysis:}

Let $s \in L^{2}(\mathbf{R})$ be an asymptotic locally monochromatic signal, and let $Z_{s}(t)$ be its analytic signal. Set:

$$
Z_{s}(t)=A_{s}(t) \exp \left(i \phi_{s}(t)\right)
$$

Let now $g \in H^{2}(\mathbf{R})$ be an analytic analysing wavelet, one then has:

$$
T^{\lambda}(b, a)=<s, \varepsilon_{\lambda} g_{(b, a)}>=\frac{1}{2}<Z_{s}, \varepsilon_{\lambda} g_{(b, a)}>
$$

Notice that the analysing wavelet being assumed to be progressive, it automatically removes in a smooth way the negative frequencies of $s(t)$, and directly works with the analytic signal $Z_{s}(t)$, as expressed by eq. $(I V-2)$. The smoothness of such a filtering (consequence of the smothness of $\hat{g}$ ) will imply a greater numerical precision than the one obtained by direct techniques involving Hilbert transform. Assume now that the analysing wavelet $g(t)$ is itself asymptotic, and set:

$$
g(t)=A_{g}(t) \exp \left(i \phi_{g}(t)\right)
$$

then all the dilated and translated wavelets $g_{(b, a)}(t)$ are asymptotic too (since they are of constant shape), and $T^{\lambda}(b, a)$ takes the form of a rapidly oscillating integral:

$$
T^{\lambda}(b, a)=\frac{1}{2 a} \int_{-\infty}^{+\infty} A_{s}(t) \overline{A_{g}\left(\frac{t-b}{a}\right)} e^{i\left[\phi_{s}(t)-\lambda t-\phi_{g}\left(\frac{t-b}{a}\right)\right]} d t
$$


the essential contribution to which is provided by the stationary points (see appendix) of the argument of the integrand, i.e. the points $t_{s}$ such that:

$$
\phi_{s}^{\prime}\left(t_{s}\right)=\lambda+\frac{1}{a} \phi_{g}^{\prime}\left(\frac{t-b}{a}\right)
$$

Note that any stationary point $t_{s}$ is a function $t_{s}(b, a)$ on the half plane $\mathbf{H}$. Consider $(b, a) \in \mathbf{H}$, and let $t_{s}^{(1)}, t_{s}^{(2)}, \ldots t_{s}^{(N)}$ be the associated stationary points, assumed to be in a finite number. Then the first order approximation to $T^{\lambda}(b, a)$ decomposes into the finite sum of the contributions $T^{\lambda}\left(t_{s}^{(n)}\right)$ of these stationary points:

$$
T^{\lambda}(b, a) \approx \sum_{n=1}^{n=N} T^{\lambda}\left(t_{s}^{(n)}\right)
$$

Set for simplicity:

$$
\Phi_{(b, a)}(t)=\phi_{s}(t)-\lambda t-\phi_{g}\left(\frac{t-b}{a}\right)
$$

If there exists an integral number $k \geq 1$ such that

$$
\left[\frac{d^{k} \Phi_{(b, a)}}{d t^{k}}\right]\left(t_{s}^{(n)}\right)=0
$$

and

$$
\left[\frac{d^{k+1} \Phi_{(b, a)}}{d t^{k+1}}\right]\left(t_{s}^{(n)}\right) \neq 0
$$

then the stationary phase argument provides an explicit formula for the contribution of the stationary point $t_{s}^{(n)}$ :

$$
T^{\lambda}\left(t_{s}^{(n)}\right)=C_{k} \frac{Z_{s}\left(t_{s}^{(n)}\right) \overline{g\left(\frac{t_{s}^{(n)}-b}{a}\right)} e^{-i \lambda t_{s}^{(n)}}}{\operatorname{Corr}_{k}(b, a)}
$$

where:

$$
\operatorname{Corr}_{k}(b, a)=a\left|\left[\frac{d^{k+1} \Phi_{(b, a)}}{d t^{k+1}}\right]\left(t_{s}^{(n)}\right)\right|^{\frac{1}{k+1}} e^{-i \frac{\pi}{2(k+1)} \operatorname{Sgn}\left[\frac{d^{k+1} \Phi(b, a)}{d t^{k+1}}\right]\left(t_{s}^{(n)}\right)}
$$

for some constant $C_{k}$. In particular, $C_{1}=\sqrt{\frac{\pi}{2}}$ and $C_{2}=\frac{1}{2} \Gamma\left(\frac{4}{3}\right) 6^{\frac{1}{3}}$.

It is interesting to notice that such an expression involves the evaluation of the analytic signal only at the stationary points. This property will be crucial in the following.

Using the same techniques, one can also get an explicit expression for the first corrective term in the asymptotic development of $T^{\lambda}(b, a)$, involving the first derivatives of the amplitudes of the signal and the translated and dilated wavelets. This term is easily seen 
to vanish in the case $k=1$, which shows the strongness of the approximation. The next corrective term (involving second derivatives of the amplitudes) is in general nonzero.

From now on, let us assume that to each point in the domain of study $\Omega \subset \mathbf{H}$ is associated a unique stationary point $t_{s}(b, a)$, such that moreover $\Phi_{(b, a)}^{\prime \prime}\left(t_{s}\right) \neq 0$ (i.e. $\left.k=1\right)$. This assumption essentially means that the components of the considered composite signal of asymptotic type do not interact, and that one can always restrict to an $\Omega$ domain in which the wavelet coefficients of all but one component are negligible. $T^{\lambda}(b, a)$ thus takes the simple form:

$$
T^{\lambda}(b, a) \approx \sqrt{\frac{\pi}{2}} \frac{e^{i \frac{\pi}{4} \operatorname{Sgn}\left[\Phi_{(b, a)}^{\prime \prime}\left(t_{s}\right)\right]}}{\sqrt{\left|\Phi_{(b, a)}^{\prime \prime}\left(t_{s}\right)\right|}} Z_{s}\left(t_{s}\right) e^{-i \lambda t_{s}} \frac{1}{a} g\left(\frac{t_{s}-b}{a}\right)
$$

On such an expression, it clearly appears that some particular sets of points will play an important role in the understanding of the wavelet transform. These sets of points are basically the points $(b, a) \in \Omega$ for which $t_{s}(b, a)=b$ (ridge of the transform) or $t_{s}(b, a)=C^{s t}$ (wavelet curves).

\section{The Ridge of the Transform:}

The ridge is defined to be the set of points $(b, a) \in \Omega$ such that $t_{s}(b, a)=b$. It immediatly follows from the definition of stationary points that on the ridge:

$$
a=a_{r}(b)=\frac{\phi_{g}^{\prime}(0)}{\phi_{s}^{\prime}(b)-\lambda}
$$

so that the ridge is a curve $\mathcal{R}=\left\{(b, a) \in \Omega ; a=a_{r}(b)\right\} \subset \Omega$ in the domain $\Omega$, from which one easily recovers the frequency modulation law. Moreover, eq. $(I V-12)$ simplifies on the ridge to:

$$
T^{\lambda}\left(b, a_{r}(b)\right) \approx \sqrt{\frac{\pi}{2}} \frac{e^{i \frac{\pi}{4} \operatorname{Sgn}\left[\Phi_{\left(b, a_{r}(b)\right)}^{\prime \prime}(b)\right]}}{\sqrt{a_{r}(b)^{2}\left|\Phi_{\left(b, a_{r}(b)\right)}^{\prime \prime}(b)\right|}} e^{-i \lambda b} \overline{g(0)} Z_{s}(b)
$$

The restriction of the wavelet transform of $s(t)$ to the associated ridge gives, up to a corrective function of $\mathrm{b}$, the analytic signal $Z_{s}(t)$ of $s(t)$. To evaluate this corrective function, note that owing to eq. $(I V-13)$, for any $(b, a) \in \Omega$ :

$$
\Phi_{(b, a)}^{\prime \prime}(t)=-\frac{a_{r}^{\prime}(t) \phi_{g}^{\prime}(0)}{a_{r}(t)^{2}}-\frac{1}{a^{2}} \phi_{g}^{\prime \prime}\left(\frac{t-b}{a}\right)
$$

and then:

$$
\Phi_{\left(b, a_{r}(b)\right)}(b)=-\frac{1}{a_{r}(b)^{2}}\left[a_{r}^{\prime}(b) \phi_{g}^{\prime}(0)+\phi_{g}^{\prime \prime}(0)\right]
$$


The correction function then depends on the analysing wavelet, and on the analysed signal only through the associated ridge. Once the ridge is known, one is then able to recover directly $A_{s}(b)$ from the wavelet coefficients $T^{\lambda}(b, a)$.

The restriction of the wavelet transform to its ridge is called the skeleton of the transform. The knowledge of the ridge and the skeleton on $\Omega$ is sufficient to characterize the transform on $\Omega$. It is then natural to introduce the corresponding skeleton operator $\mathcal{F}_{0}^{\mathcal{R}}: L^{2}(\mathbf{R}) \rightarrow L^{2}(\mathbf{R})$, defined by:

$$
\left[\mathcal{F}_{0}^{\mathcal{R}} . s\right](t)=\sqrt{\frac{2}{\pi}} \frac{\sqrt{a^{2}\left|\Phi_{\left(b, a_{r}(b)\right)}^{\prime \prime}(b)\right|}}{\overline{g(0)}} e^{i \lambda b} e^{-i \frac{\pi}{4} \operatorname{Sgn}\left[\Phi_{\left(b, a_{r}(b)\right.}^{\prime \prime}(b) \mid\right.} T^{\lambda}\left(b, a_{r}(b)\right) \quad(I V-17)
$$

and whose action is to select the content of the signal localised around the ridge.

Remark: The phase shift $\pm \frac{\pi}{4}$ is constant here, since we have assumed that for any $(b, a) \in \Omega, \Phi_{(b, a)}^{\prime \prime}\left(t_{s}\right) \neq 0$, so that the sign of $\Phi_{(b, a)}^{\prime \prime}\left(t_{s}\right)$ does not change on $\Omega$.

\section{The wavelet curves:}

Consider $\left(b_{0}, a_{r}\left(b_{0}\right)\right) \in \Omega$. The wavelet curve through $\left(b_{0}, a_{r}\left(b_{0}\right)\right)$ is defined to be the connected component of $\left(b_{0}, a_{r}\left(b_{0}\right)\right)$ of the set of points $(b, a) \in \Omega$ such that $t_{s}(b, a)=b_{0}$. Let $(b, a) \in \Omega$ be such a point; this implies that:

$$
\phi_{g}^{\prime}\left(\frac{b_{0}-b}{a}\right)=\frac{a}{a_{r}\left(b_{0}\right)} \phi_{g}^{\prime}(0)
$$

The wavelet curves are then uniquely determined by the analysing wavelet. For instance, in the case of fixed-frequency wavelets, $\phi_{g}^{\prime}(0)=\omega_{0}$, and the wavelet curve through $\left(b_{0}, a_{r}\left(b_{0}\right)\right)$ is the line $a=a_{r}\left(b_{0}\right)$; this property is a characteristic property of the fixed-frequency analysing wavelets.

Restricting the wavelet transform to a given wavelet curve yields:

$$
T^{\lambda}(b, a) \approx \sqrt{\frac{\pi}{2}} \frac{e^{i \frac{\pi}{4} S g n\left[\Phi_{(b, a)}^{\prime \prime}\left(b_{0}\right)\right]}}{\sqrt{a^{2}\left|\Phi_{(b, a)}^{\prime \prime}\left(b_{0}\right)\right|}} Z_{s}\left(b_{0}\right) e^{-i \lambda b_{0}} \overline{g\left(\frac{b_{0}-b}{a}\right)}
$$

so that the behaviour of the wavelet transform restricted to a given wavelet curve is governed by that of the corresponding dilated and translated wavelets (the dilation parameter $a$ being a function of the translation parameter b). Once more, $\left|T^{\lambda}(b, a)\right|$ is obtained from $\overline{g\left(\frac{b_{0}-b}{a}\right)}$ via a perturbation by the correction function $\sqrt{a^{2}\left|\Phi_{(b, a)}^{\prime \prime}\left(b_{0}\right)\right|}$.

It is worth noticing that one now has a nice geometrical picture of the wavelet transform in the time-scale half plane: when restricted to the ridge, it is essentially determined by the analytic signal of the analysed signal, and when restricted to a given wavelet curve, it is essentially determined by the wavelets, in both cases up to a corrective function, completely determined by the ridge and the analysing wavelet. This is the so-called factorisation property of the transform, that will be frequently used in the sequel. 


\section{Ridge extraction:}

The next question is that of the extraction of the ridge from the $T^{\lambda}(b, a)$ coefficients. The most usual and natural information comes from the squared modulus of the coefficients, interpreted as an energy density in the time-scale half-plane $\mathbf{H}$. The analysing wavelet being assumed to be maximum at $t=0$, eq. $(I V-12)$ shows that $\left|T^{\lambda}(b, a)\right|$ is locally maximum at $b=t_{s}$, if one neglects the influence of the corrective function in the denominator of eq. $(I V-14)$. Taking this corrective function into account shows that the maximality of $T^{\lambda}(b, a)$ at $b=t_{s}$ only holds in an approximate sense, which is exact if and only if the ridge $a_{r}(b)$ is a linear function of $b$, i.e. for hyperbolically frequency modulated signals, and $\phi_{g}^{\prime \prime}(0)=0$.

The ridge of the transform can be extracted in a much more precise way from the phase of the wavelet coefficients. Let:

$$
\Psi^{\lambda}(b, a)=\arg \left[T^{\lambda}(b, a)\right]
$$

Then by definition of $t_{s}(b, a)$ :

$$
\left.\frac{\partial \Psi^{\lambda}(b, a)}{\partial a}=-\frac{t_{s}-b}{a^{2}} \Phi_{(b, a)}\right)^{\prime}\left(\frac{t_{s}-b}{a}\right)
$$

and then vanishes on the intersection of the lines $b=C^{s t}$ and the ridge. This gives an easy way to extract numerically the ridge of the wavelet transform.

Moreover, the argument of $T^{\lambda}(b, a)$, restricted to a given wavelet curve, has the following other property:

$$
\begin{gathered}
{\left[\frac{\partial \Psi^{\lambda}(b, a)}{\partial b}\right]_{t_{s}(b, a)=b_{0}}=\frac{1}{a} \phi_{g}^{\prime}\left(\frac{t_{s}-b}{a}\right)+\left[\frac{\partial a}{\partial b}\right] \frac{t_{s}-b}{a^{2}} \phi_{g}^{\prime}\left(\frac{t_{s}-b}{a}\right)} \\
=\frac{\phi_{g}^{\prime}(0)}{a} \text { on the intersection with the ridge }
\end{gathered}
$$

Hence the instantaneous frequency of the restriction of the wavelet transform to a fixed wavelet curve equals the central frequency of the dilated wavelet at the intersection with the ridge. This provides another algorithm for the ridge extraction, which will be discussed in section VI.

Remark: Note that in the case of a fixed-frequency analysing wavelet, such a property does not allow the extraction of the ridge. Indeed, in that case, $t_{s}(b, a)=b_{0}$ is equivalent to $a=a_{r}\left(b_{0}\right)$, and one has that $\frac{\partial \Psi^{\lambda}}{\partial b}(b, a)=\frac{\phi_{g}^{\prime}(0)}{a}$ everywhere. This in particular illustrates the fact that the extraction algorithms described here are based on necessary conditions rather than necessary and sufficient conditions, and that the ridge is a subset of the set of points in $\Omega$ such that $(V I-23)$ holds, and not the whole set of such points.

\section{IV-2: Gabor Analysis:}


We keep the same notations than in the section IV-1, and we will consider only the points $(b, \omega) \in \Omega \subset \mathbf{H}$ such that $g_{(b, \omega)} \in H^{2}(\mathbf{R})$, or at least that the Fourier transform of $g_{(b, \omega)}$ is numerically negligible at negative frequencies. Under such an assumption, one has that:

$$
G(b, \omega)=<s, g_{(b, \omega)}>=\frac{1}{2}<Z_{s}, g_{(b, \omega)}>
$$

Assuming that all the $g_{(b, \omega)}$ functions under consideration are asymptotic, the oscillating integral defining $G(b, \omega)$ :

$$
G(b, \omega)=\int_{-\infty}^{+\infty} A_{s}(t) \overline{A_{g}(t-b)} e^{i\left[\phi_{s}(t)-\phi_{g}(t-b)-\omega(t-b)\right]} d t
$$

can be approximated by the stationary phase method: the essential contribution to eq. $(I V-26)$ is provided by the stationary points of the phase of the integrand, i.e. by the points $t_{s}(b, \omega)$ such that:

$$
\phi_{s}^{\prime}\left(t_{s}\right)=\phi_{g}\left(t_{s}-b\right)+\omega
$$

As in the previous case, we restrict to a region $\Omega \subset \mathbf{H}$ such that to each $(b, \omega) \in \Omega$ is associated a unique stationary point $t_{s}(b, \omega)$, which is a first order stationary point. One then has the following approximation for the Gabor transform:

$$
G(b, \omega) \approx \sqrt{\frac{\pi}{2}} \frac{e^{i \frac{\pi}{4} \operatorname{Sgn}\left[\Phi_{(b, \omega)}^{\prime \prime}\left(t_{s}\right)\right]}}{\sqrt{\left|\Phi_{(b, \omega)}^{\prime \prime}\left(t_{s}\right)\right|}} Z_{s}\left(t_{s}\right) e^{-i \omega\left(t_{s}-b\right)} \overline{g\left(t_{s}-b\right)}
$$

where one has set:

$$
\Phi_{(b, \omega)}(t)=\phi_{s}(t)-\phi_{g}(t-b)-\omega(t-b)
$$

As in the case of the wavelet transform, one can introduce the notions of ridge and skeleton of the transform, and that of Gabor curves.

\section{The ridge of the transform:}

The ridge is defined to be the set of points $(b, \omega) \in \Omega$ such that $t_{s}(b, \omega)=b$. By definition of the stationary points, one has that, on the ridge:

$$
\omega=\omega_{r}(b)=\phi_{s}^{\prime}(b)-\phi_{g}^{\prime}(0)
$$

so that the ridge gives a direct information on the instantaneous frequency modulation law of the signal. Moreover, eq. $(I V-28)$ reduces to:

$$
G\left(b, \omega_{r}(b)\right) \approx \sqrt{\frac{\pi}{2}} \frac{e^{i \frac{\pi}{4} \operatorname{Sgn}\left[\Phi_{\left(b, \omega_{r}(b)\right)}^{\prime \prime}(b)\right]}}{\sqrt{\left|\Phi_{\left(b, \omega_{r}(b)\right)}^{\prime \prime}(b)\right|}} \overline{g(0)} Z_{s}(b)
$$


Note that:

$$
\Phi_{\left(b, \omega_{r}(b)\right)}^{\prime \prime}(b)=\omega_{r}^{\prime}(b)-\phi_{g}^{\prime \prime}(0)
$$

The correction function appearing in the denominator of eq. $(I V-31)$ is then completely determined by the analysing function and the ridge of the transform, and the Gabor analogue of the skeleton operator (see eq. $(I V-20)$ ) can still be introduced.

The restriction of the transform to its ridge is called the skeleton of the Gabor transform.

\section{The Gabor curves:}

Let $\left(b_{0}, \omega_{r}\left(b_{0}\right)\right) \in \Omega$ be a point on the ridge of the Gabor transform of $s(t)$. The Gabor curve through $\left(b_{0}, \omega_{r}\left(b_{0}\right)\right) \in \Omega$ is the connected component of $\left(b_{0}, \omega_{r}\left(b_{0}\right)\right) \in \Omega$ of the set of points $(b, \omega) \in \Omega$ such that $t_{s}(b, \omega)=b_{0}$. Let $(b, \omega) \in \Omega$ be such a point: this implies that:

$$
\phi_{g}^{\prime}\left(b_{0}-b\right)=\phi_{g}^{\prime}(0)+\omega\left(b_{0}-b\right)
$$

which means that such curves are completely determined by the analysing function. The restriction of $G(b, \omega)$ to the Gabor curve associated with $b_{0}$ reads:

$$
G(b, \omega) \approx \sqrt{\frac{\pi}{2}} \frac{e^{i \frac{\pi}{4} \operatorname{Sgn}\left[\Phi_{(b, \omega)}^{\prime \prime}\left(b_{0}\right)\right]}}{\sqrt{\left|\Phi_{(b, \omega)}^{\prime \prime}\left(b_{0}\right)\right|}} Z_{s}\left(b_{0}\right) e^{-i \omega\left(b-b_{0}\right)} \overline{g\left(b_{0}-b\right)}
$$

which shows that the behaviour of the Gabor coefficients restricted to a Gabor curve is essentially governed by that of the Gabor function $e^{-i \omega\left(b-b_{0}\right)} \overline{g\left(b_{0}-b\right)}$.

\section{Ridge extraction:}

The ridge can be extracted from the $G(b, \omega)$ coefficients in an approximate way by the study of the energy localisation in the time-frequency plane, i.e. by the determination of the local maxima of the modulus $|G(b, \omega)|$. But as in the case of wavelet analysis, the study of:

$$
\Psi(b, \omega)=\arg (G(b, \omega))
$$

allows a much more precise determination of the ridge. Indeed, a straightforward calculation yields:

$$
\begin{gathered}
{\left[\frac{\partial \Psi(b, \omega)}{\partial \omega}\right]_{b=C^{s t}}=-\left(t_{s}-b\right)} \\
=0 \text { on the ridge }
\end{gathered}
$$

which is the Gabor analogue of eq. $(I V-18)$. The ridge of the Gabor transform is then easy to determine numerically. $|G(b, \omega)|$ is obtained from $\left|g\left(b_{0}-b\right)\right|$ by a perturbation by $\sqrt{\left|\Phi_{(b, \omega)}^{\prime \prime}\left(b_{0}\right)\right|}$, and $\arg G(b, \omega)$ has the following property, when restricted to a fixed Gabor curve:

$$
\left[\frac{\partial \Psi(b, \omega)}{\partial b}\right]_{t_{s}(b, \omega)=b_{0}}=\omega+\phi_{g}^{\prime}\left(b_{0}-b\right)-\left[\frac{\partial \omega}{\partial b}\right]\left(b_{0}-b\right)
$$




$$
=\omega+\phi_{g}^{\prime}(0) \text { on the intersection with the ridge }
$$

$(I V-39)$

The right hand side is nothing but the proper frequency of the Gabor function $g_{(b, \omega)}(t)$. This gives another way of extracting the instantaneous frequency, which will be discussed in the next chapter. 


\section{V: NON-ASYMPTOTIC ANALYSING FUNCTION:}

It must be noticed that the asymptotic assumption made on the analysing functions in the last section is not very natural either in the wavelet or in the Gabor philosophy, in the sense that the frequency localisation of these functions is enforced, while the time localisation is destroyed. This has moreover a bad consequence on the numerical point of view, in the sense that the computational time gets increased.

In the case where the analysing functions have a Gaussian amplitude, it is however possible to slightly generalize the stationary phase calculus to non asymptotic wavelets (or Gabor curves), and still asymptotic signals. The notions of ridge and wavelet and Gabor curves can still be introduced, and have the same intuitive meaning. The only changes concern the explicit expressions of the coefficients, and the ridge extraction algorithms.

\section{V-1: Wavelet Analysis:}

Consider an analytic analysing wavelet $g \in H^{2}(\mathbf{R})$, such that its amplitude $A_{g}(t)$ is a Gaussian function, which can be supposed without loss of generality to be normal. By a slight modification of the stationary phase principle (see appendix), eq. ( $I V-4)$ can now be approximated by:

$$
T^{\lambda}(b, a) \approx \frac{A_{s}\left(t_{s}\right) e^{i \Phi_{(b, a)}\left(t_{s}\right)}}{2 a} \int_{-\infty}^{+\infty} A_{g}\left(\frac{t-b}{a}\right) e^{i \frac{\left(t-t_{s}\right)^{2}}{2} \Phi_{(b, a)}^{\prime \prime}\left(t_{s}\right)} d t
$$

which, up to a rotation in the complex plane, reduces to the computation of a Gaussian integral. Here, $t_{s}=t_{s}(b, a)$ is still defined by eq. $(I V-5)$. A straightforward computation then yields:

$$
\begin{aligned}
& T^{\lambda}(b, a)=\left|T^{\lambda}(b, a)\right| e^{i \Psi^{\lambda}(b, a)} \\
& \left|T^{\lambda}(b, a)\right| \approx \sqrt{\frac{\pi}{2}} \frac{A_{s}\left(t_{s}\right) e^{-\frac{1}{2} \frac{\left(t_{s}-b\right)^{2} \Phi_{(b, a)}^{\prime \prime}\left(t_{s}\right)^{2}}{\Phi_{(b, a)}^{\prime \prime}\left(t_{s}\right)^{2}+\frac{1}{a^{4}}}}}{\left[1+a^{4} \Phi_{(b, a)}^{\prime \prime}\left(t_{s}\right)^{2}\right]^{\frac{1}{4}}} \\
& \Psi^{\lambda}(b, a) \approx \phi_{s}\left(t_{s}\right)-\lambda t_{s}-\phi_{g}\left(\frac{t_{s}-b}{a}\right)+\frac{1}{2} \frac{\left(t_{s}-b\right)^{2} \Phi_{(b, a)}^{\prime \prime}\left(t_{s}\right)}{1+a^{4} \Phi_{(b, a)}^{\prime \prime}\left(t_{s}\right)^{2}}+\frac{1}{2} \arctan \left[a^{2} \Phi_{(b, a)}^{\prime \prime}\left(t_{s}\right)\right] \\
& (V-2-a) \\
& (V-2-b) \\
& (V-2-c)
\end{aligned}
$$

Contrary to the case studied in section IV, the first corrective term in the asymptotic development, proportional to $A_{s}^{\prime}\left(t_{s}\right)$ is not identically zero in $\Omega$. This term reads:

$$
T_{(1)}^{\lambda}(b, a)=\frac{A_{s}^{\prime}\left(t_{s}\right)}{2 a} e^{i \Phi_{(b, a)}\left(t_{s}\right)} \int_{-\infty}^{+\infty}\left(t-t_{s}\right) A_{g}\left(\frac{t-b}{a}\right) e^{i \frac{\left(t-t_{s}\right)^{2}}{2} \Phi_{(b, a)}^{\prime \prime}\left(t_{s}\right)} d t \quad(V-3)
$$

and can be shown to equal:

$$
T_{(1)}^{\lambda}(b, a)=\left|T_{(1)}^{\lambda}(b, a)\right| e^{i \Psi_{(1)}^{\lambda}(b, a)} \quad(V-4-a)
$$




$$
\begin{aligned}
& \left|T_{(1)}^{\lambda}(b, a)\right|=\sqrt{\frac{\pi}{2}} \frac{A_{s}^{\prime}\left(t_{s}\right) e^{-\frac{1}{2} \frac{\left(t_{s}-b\right)^{2} \Phi_{(b, a)}^{\prime \prime}\left(t_{s}\right)^{2}}{\Phi_{(b, a)}^{\prime \prime}\left(t_{s}\right)^{2}+\frac{1}{a^{4}}}}}{\left[1+a^{4} \Phi_{(b, a)}^{\prime \prime}\left(t_{s}\right)^{2}\right]^{\frac{3}{4}}}\left|t_{s}-b\right| \quad(V-4-b) \\
& \Psi_{(1)}^{\lambda}(b, a)=\phi_{s}\left(t_{s}\right)-\lambda t_{s}-\phi_{g}\left(\frac{t_{s}-b}{a}\right)+\frac{1}{2} \frac{\left(t_{s}-b\right)^{2} \Phi_{(b, a)}^{\prime \prime}\left(t_{s}\right)}{1+a^{4} \Phi_{(b, a)}^{\prime \prime}\left(t_{s}\right)^{2}}+\frac{3}{2} \arctan \left[a^{2} \Phi_{(b, a)}^{\prime \prime}\left(t_{s}\right)\right]
\end{aligned}
$$

Note that $T_{(1)}^{\lambda}(b, a)$ is proportional to $\left|t_{s}-b\right|$, which is not the case of the second corrective term $T_{(2)}^{\lambda}(b, a)$ of the asymptotic development.

\section{Ridge and skeleton:}

Define the ridge of the transform to be the set of points $(b, a) \in \Omega$ such that $t_{s}(b, a)=b$. It immediately follows from the definition of stationary points that eq. $(I V-14)$ is still valid, so that the knowledge of the ridge of the wavelet transform is enough to give a direct access to the frequency modulation law.

The skeleton of the transform, i.e. the restriction of the wavelet transform to the associated ridge has a slightly different form than that given by eq. $(I V-15)$ :

$$
T^{\lambda}\left(b, a_{r}(b)\right) \approx \sqrt{\frac{\pi}{2}} \frac{e^{\frac{i}{2} \arctan \left[a_{r}(b)^{2} \Phi_{\left(b, a_{r}(b)\right)}^{\prime \prime}(b)\right]}}{\left[1+a^{4} \Phi_{(b, a)}^{\prime \prime}\left(t_{s}\right)^{2}\right]^{\frac{1}{4}}} e^{-i \lambda b-i \phi_{g}(0)} Z_{s}(b)\left|t_{s}-b\right|
$$

while from eq. $(V-4-b)$ :

$$
T_{(1)}^{\lambda}\left(b, a_{r}(b)\right)=0
$$

Finally, eq. $(I V-16)$ still holds. Thus, the skeleton of the wavelet transform of the asymptotic signal $s \in L^{2}(\mathbf{R})$ gives, up to a corrective function which is completely determined by the analysing wavelet and the ridge itself,the analytic signal $Z_{s}(t)$ of $s(t)$. Eq. $(V-6)$ also indicates that the first corrective term to this approximation vanishes identically.

\section{Wavelet curves and ridge extraction:}

The extraction of the ridge of the transform from the zeroes of $\left[\frac{\partial \Psi^{\lambda}}{\partial a}\right]_{b=b_{0}}$ is no longer valid. Indeed, it is not difficult to check that $\left[\frac{\partial \Psi^{\lambda}}{\partial a}\right]_{b=b_{0}}$ involves a (in general nonzero) term proportional to $\left[\frac{\partial t_{s}}{\partial a}\right]_{b=b_{0}}$.

Consider now the wavelet curves, as defined in section IV-1, i.e. essentially as the sets of points $(b, a) \in \Omega$ with constant stationary point. The restriction of the wavelet transform to such a curve is essentially governed by the corresponding translated and dilated wavelets (its expression is obtained by setting $t_{s}=b_{0}$ in eq. $(V-4)$, for some fixed $b_{0}$. Note that the phase of $T^{\lambda}(b, a)$ is now perturbed by the contribution of the modulus of the wavelet. 
Nevertheless, it can be a useful quantity for the extraction of the ridge, since on a fixed wavelet curve:

$$
\left[\frac{d \Psi^{\lambda}}{d b}\right]_{t_{s}(b, a)=b_{0}}=\frac{1}{a}\left[\phi_{g}^{\prime}(0)+\frac{1}{2} \frac{\phi_{g}^{\prime \prime \prime}(0)}{1+\left[\phi_{g}(0) a_{r}^{\prime}(b)+\phi_{g}^{\prime \prime}(0)\right]^{2}}\right]
$$

on the intersection with the ridge, so that for any analysing wavelet such that its central frequency is an inflection point (i.e. $\left.\phi_{g}^{\prime \prime \prime}(0)=0\right)$, eq. $(I V-21)$ is still valid, providing an algorithm for the extraction of the ridge.

\section{V-2: Gabor Analysis:}

Assume that the window is a function of Gaussian type, once more assumed to be normal. The approximation:

$$
G(b, \omega) \approx \frac{1}{2} A_{s}\left(t_{s}\right) e^{i \Phi_{(b, \omega)}\left(t_{s}\right)} \int_{-\infty}^{+\infty} e^{-\frac{1}{2}(t-b)^{2}} e^{i\left(t-t_{s}\right)^{2} \Phi_{(b, \omega)}^{\prime \prime}\left(t_{s}\right)} d t
$$

leads to:

$$
\begin{aligned}
& G(b, \omega)=|G(b, \omega)| e^{i \Psi(b, \omega)} \\
& (V-9-a) \\
& |G(b, \omega)| \approx \sqrt{\frac{\pi}{2}} \frac{A_{s}\left(t_{s}\right) e^{-\frac{1}{2} \frac{\left(t_{s}-b\right)^{2} \Phi(b, \omega)^{\prime \prime}\left(t_{s}\right)^{2}}{1+\Phi_{(b, \omega)}^{\prime \prime}\left(t_{s}\right)^{2}}}}{\left[1+\Phi_{(b, \omega)}^{\prime \prime}\left(t_{s}\right)^{2}\right]^{\frac{1}{4}}} \\
& \Psi(b, \omega) \approx \Phi_{(b, \omega)}\left(t_{s}\right)+\frac{1}{2} \frac{\left(t_{s}-b\right)^{2} \Phi_{(b, \omega)}^{\prime \prime}\left(t_{s}\right)}{1+\Phi_{(b, \omega)}^{\prime \prime}\left(t_{s}\right)^{2}}+\frac{1}{2} \arctan \left[\Phi_{(b, \omega)}^{\prime \prime}\left(t_{s}\right)^{2}\right] \quad(V-9-c)
\end{aligned}
$$

Moreover, one easily checks that, as in the wavelet case, the first corrective term in the asymptotic development is proportional to $\left|t_{s}-b\right|$.

\section{Ridge and skeleton:}

Still defining the ridge of the Gabor transform by $t_{s}(b, \omega)=b$, eq. $(I V-30)$ remains valid. The skeleton of the transform is now given by:

$$
G\left(b, \omega_{r}(b)\right) \approx \sqrt{\frac{\pi}{2}} \frac{e^{\frac{i}{2} \arctan \Phi_{\left(b, \omega_{r}(b)\right)}^{\prime \prime}(b)}}{\left[1+\Phi_{\left(b, \omega_{r}(b)\right)}^{\prime \prime}(b)^{2}\right]^{\frac{1}{4}}} \overline{g(0)} Z_{s}(b)
$$

and reproduces the analytic signal $Z_{s}(t)$, up to the corrective function:

$$
\sqrt{\frac{\pi}{2}} \frac{e^{\frac{i}{2} \arctan \Phi_{\left(b, \omega_{r}(b)\right)}^{\prime \prime}(b)}}{\left[1+\Phi_{\left(b, \omega_{r}(b)\right)}^{\prime \prime}(b)^{2}\right]^{\frac{1}{4}}} \overline{g(0)}
$$


which is completely determined by the ridge.

\section{Gabor curves and ridge extraction:}

Defining the Gabor curves by eq. $(I V-36)$, it is not difficult to see that on a given Gabor curve, eq. (IV-39) becomes:

$$
\left[\frac{d \Psi}{d b}\right]_{t_{s}(b, \omega)=b_{0}}=\omega+\phi_{g}^{\prime}(0)+\frac{1}{2} \frac{\phi_{g}^{\prime \prime \prime}(0)}{1+\left[\omega_{r}^{\prime}(b)+\phi_{g}^{\prime \prime}(0)\right]^{2}}
$$

on the intersection with the ridge, leading to a simple algorithm for the extraction of the ridge in cases where $\phi_{g}^{\prime \prime \prime}(0)=0$. 


\section{VI: RIDGE AND SKELETON EXTRACTIONS:}

We now turn to numerical applications of the asymptotic formulas described in the previous sections. As stressed in the introduction, we are interested in the study of asymptotic signals, but also of composite signals of asymptotic type, defined as linear combinations of asymptotic signals. Clearly, such signals need not be asymptotic themselves, because of interference phenomena. We will consider in the same footing wavelet and Gabor analysis, which have been shown to have the same behaviour. In the wavelet case, we will only describe the $\lambda=0$ case for simplicity, keeping the possibility of introducing the $\lambda$ parameter to enforce the asymptotic character of the signal.

Throughout this section, we will only use for numerical computations the so-called Morlet wavelet:

$$
g(t)=e^{-\frac{t^{2}}{2}} e^{i \omega_{0} t}
$$

Although the Morlet wavelet is not rigorously admissible, $\omega_{0}$ can be chosen sufficiently large so that $\hat{g}(0)$ is numerically negligible. Under such an assumption, $g(t)$ can be considered as a numerically progressive wavelet, so that eqs. $(I V-2)$ and $(I V-25)$ hold. Notice that in that case, the wavelet curves are nothing but the $a=C^{s t}$ curves, and the Gabor curves are the $\omega=C^{s t}$ curves, which makes the numerical implementation simpler.

\section{VI-1: An algorithm for ridge extraction: non-interacting ridges:}

Let us first consider the case of non-interacting components, that is the case where the asymptotic components of the signal live in different regions of the time-frequency half-plane. Because of the good localisation properties of both $g$ and $\hat{g}$, we are then led to the case of asymptotic signals. We will say that in such a case, the individual ridges are non-interacting. Our aim is to extract numerically an individual ridge from the (wavelet or Gabor) transform, but also to avoid computing the whole transform by restraining the computation to a neighborhood of the ridge. Let us first give a simple example:

\section{An intuitive view of the problem:}

Let us look at the simplest case of a monochromatic signal: let

$$
s(t)=\exp \left(i \omega_{s} t\right)
$$

Its wavelet transform with respect to the progressive analysing wavelet $g$ reads:

$$
T(b, a)=|T(b, a)| e^{i \Psi(b, a)}=\overline{\hat{g}}\left(a \omega_{s}\right) \exp \left(i \omega_{s} b\right)
$$

Clearly, the ridge of the wavelet transform of such a signal is given by:

$$
a_{r}=\frac{\omega_{0}}{\omega_{s}}
$$


Moreover, $a_{r}$ can obviously be reached iteratively as follows: let $a_{0}$ be a primary value of the dilation parameter. Compute:

$$
\omega_{1}=\frac{\partial \Psi\left(b, a_{0}\right)}{\partial b}
$$

and set:

$$
a_{1}=\frac{\omega_{0}}{\omega_{1}}
$$

Clearly $a_{1}=a_{r}$, and:

$$
\frac{\omega_{0}}{\frac{\partial \Psi\left(b, a_{r}\right)}{\partial b}}=a_{r}
$$

The ridge is then obtained as a fixed point of an iterative scheme in such a particular case.

\section{The algorithm:}

Let us now generalise the approach described above, focusing on the algorithmic aspect. Consider the case of discrete sequences: let $T_{e}=\frac{1}{\nu_{e}}$ be the sampling period, consider the wavelet transform $T_{a}(k)=T\left(k T_{e}, a\right)$ of $s_{k}=s\left(k T_{e}\right)$ for a fixed value of the dilation parameter, and $\Psi_{a}(k)$ its argument. Denote by $D_{b}$ an estimator of a discrete differentiation with respect to $b$. Then, the following algorithm allows the construction of a trajectory $a_{r}\left(k T_{e}\right)$ in the $\Omega$ domain such that:

$$
D_{b} . \Psi_{a}(k)=\frac{\omega_{0}}{a}
$$

The algorithm can be described in a similar way than above: let $a_{0}\left(t_{0}\right)$ be an initial estimate of $a_{r}\left(t_{0}\right)$. Then the estimates for $a_{r}\left(t_{0}+k T_{e}\right)$ are given by:

$$
\begin{gathered}
a_{i+1}\left(t_{0}+k T_{e}\right)=\frac{\omega_{0}}{D_{b} . \Psi_{a_{i}}\left(t_{0}+k T_{e}\right)} \\
a_{0}\left(t_{0}+(k+1) T_{e}\right)=a_{c}\left(t_{0}+k T_{e}\right)
\end{gathered}
$$

where $a_{c}$ is the "converged solution", i.e. the value $a_{c}=a_{j}$ of the dilation parameter for which one has, for some fixed positive value of the required precision $\epsilon$ (arbitrarily small fixed positive number):

$$
\left|\frac{a_{j+1}-a_{j}}{a_{j}}\right|<\epsilon
$$

In practice, such an algorithm converges very rapidly, two or three iterations being necessary for each signal sample. This in particular means that one does not have to compute the whole wavelet transform to get an estimate of its ridge.

\section{Numerical results:}

Since the signals studied here are assumed to have non-interacting ridges, we first give the example of an academic signal $s(t)=\exp \left(i \omega_{s} t+i \alpha_{s} \ln (1+\beta t)\right)$, hyperbolically 
modulated in frequency (it is clearly asymptotic since its amplitude does not vary). Fig. VI-1-a represents the phase and the modulus of $T_{s}$, and the ridge $a=a_{r}(b)$ is represented on the phase plot. Fig. VI-1-b the frequency and amplitude modulation laws of such a signal, computed via wavelet transform. The results are in excellent agreement with the input data.

Numerical tests can also be performed on real signals, like for instance a sonar signal emitted by a bat. In Fig. VI-2-a and VI-2-b, its frequency modulation law is seen to be close to an hyperbolic one. Notice the existence in Fig. VI-2-a of an auxiliary ridge at higher frequencies, which very presumably results from an echo phenomenon during the experiment. It is quite interesting to compare the results obtained with wavelets to those obtained with standard Hilbert transform-based techniques, in the case where the previous signal is embedded in noise (here a uniform computer noise, with amplitude comparable to that of the signal itself). In that case, the standard method (Fig. VI-3-a) fails to extract correctly the frequency and amplitude modulation laws, because they use the whole timefrequency content of the input signal, while the good localisation properties of both $g$ and $\hat{g}$ allow to take into account only the subset of the time-frequency plane where the asymptotic signal is important, and thus yield much more precise results (Fig. VI-3-b), in particular for the instantaneous frequency. Moreover, wavelet and Gabor transform techniques also allow the separation of the auxiliary component of the signal, which is completely impossible with the standard techniques. Notice also that the Hilbert transform method is able to yield a precise result in that case only because the second component of the signal is much smaller in amplitude than the first one.

\section{VI-2: Interacting ridges:}

Consider now the cases where the input signal $s(t)$ is the sum of say two asymptotic signals, and that the corresponding wavelet (or Gabor) transforms are non-negligible in intersecting domains $\Omega_{1}$ and $\Omega_{2}$. This is what happens for instance in Fig. VI-4, which represents the wavelet transform of two linearly modulated cosine functions, with the same modulation rate $\alpha$. Interference phenomena cause here a strong perturbation of the phase of the wavelet transform, so that the ridge extraction algorithm provided by eq. $(I V-21)$ is no longer exact. Indeed, in Fig. VI-4, small "bubbles" regularly appear on the ridge plot (we will understand the origin of such bubbles a little later). In some particular cases, the interferences can be made negligible by a suitable choice of the analysing wavelet. For instance, with the same signal than the one studied in fig. VI-4, taking a Morlet wavelet with a much greater value of $\omega_{0}$ will yield a family of wavelets with a much better localisation in the Fourier space, so that they will be able to separate the two components. This is the case in Fig. VI-5, where the proper frequency of the Morlet wavelet has been chosen large enough to avoid interferences between the two components, and allows their separation and their extraction. Unfortunately, such a simple trick does not always work, in particular in the case of complex signals. For instance, Fig. VI-6-a and VI-6-b represent the phase of the Gabor transform of a trumpet sound and a female voice respectively, and the associated ridges. The ridge structure is in such cases so complex that it cannot be 
analysed by the methods described here, even with a particular choice of the analysing functions. This is a strong limitation of the asymptotic approximations-based algorithms described in this paper.

In some very simple and particular example, the existence of the bubbles can be identified as coming from interactions between two or more modulated component. Consider then a signal made of two spectral lines with equal amplitudes (set to 1) and pulsations $\omega_{1}$ and $\omega_{2}: s(t)=\exp \left(i \omega_{1} t\right)+\exp \left(i \omega_{2} t\right)$, to be analysed with Gabor analysis, with analysing

function $g(t)=\exp \left(-\frac{t^{2}}{2 \sigma}\right) \exp (i \omega t)$ (fixed frequency analysing function). A straightforward computation shows that $b$-derivative of the phase of $G(b, \omega)$ is given by:

$$
\frac{\partial \Psi(b, \omega)}{\partial b}=\omega^{*}+\delta \tanh (s) \frac{1+\tan ^{2}(b \delta)}{1+\tan ^{2}(b \delta) \tanh ^{2}(s)}
$$

where:

$$
\begin{gathered}
\omega^{*}=\frac{\omega_{2}+\omega_{1}}{2} \\
\delta=\frac{\omega_{2}-\omega_{1}}{2}
\end{gathered}
$$

and

$$
s=\sigma^{2}\left(\omega-\omega^{*}\right) \delta
$$

The ridge equation is then a fixed point equation on s:

$$
s=\sigma^{2} \delta^{2} \tanh (s) \frac{1+\tan ^{2}(b \delta)}{1+\tan ^{2}(b \delta) \tanh ^{2}(s)}
$$

Depending on the values of $b$ and of the parameters, the number of solutions of such a fixed point equation determines the existence of bubbles. For large values of $\sigma \delta$, i.e. for non-interacting ridges, the fixed points are close to $\omega_{1}$ and $\omega_{2}$. Otherwise, consider the case of small values of $\sigma \delta$; then whenever $\tan ^{2}(b \delta)$ is large enough, the fixed point equation has three solutions ( $s=0$ and two symmetric ones), while for small values of $\tan ^{2}(b \delta)$, only $s=0$ is possible, so that a bubble may be formed. This is illustrated in Fig. VI-7. For real signals, the bubble structure is in general more complex, as illustrated in Fig. VI-8-a and VI-8-b, showing the ridges of the Gabor transform of a clarinet sound (at different times), containing three frequency modulated components. In that example, the interaction between the different ridges increases with time, and bubbles appear.

\section{VI-3: Spectral lines estimation:}

We now restrict our interest to special kinds of signals, that is to asymptotic signals that are sums of spectral lines:

$$
s(t)=\sum s l_{i}(t)
$$

where a spectral line is of the form:

$$
s l_{i}(t)=A_{i}(t) \exp \left[i \omega_{i} t+i \delta_{i}\right]
$$


Such signals are quite interesting for instance from the acoustical point of view, since they allow to describe many vibrating systems. We will discuss here a method of estimation of the frequencies $\omega_{i}$ and the amplitudes $A_{i}(t)$, under the assumptions that all the $A_{i}(t)$ are analytic and vary slowly in the domain of study.

\section{The case of one spectral line:}

Consider first the case of a monochromatic signal of constant amplitude $A$ and pulsation $\omega_{s}$. Then clearly $T(b, a)=A \exp \left(i \omega_{s} b\right) \hat{g}\left(a \omega_{s}\right)$. Assuming that $\hat{g}$ is real, which is indeed the case for the Morlet wavelet, clearly $\frac{\partial \Psi(b, a)}{\partial b}=\omega_{s}$.

Assuming now that $A_{s}(t)$ is an analytic function of $t$ :

$$
s l(t)=A_{s}(t) \exp \left(i \omega_{s} t\right)
$$

and since the analysing wavelet is assumed to be maximal at $t=0$, consider the following asymptotic development of $T_{s l}(b, a)$ :

$$
T_{s l}(b, a)=A_{s}(b) e^{i \omega_{s} b}\left[\overline{\hat{g}}\left(a \omega_{s}\right)+\sum_{k=1}^{\infty} \frac{(-i a)^{k}}{k !} \frac{d^{k} \overline{\hat{g}}}{d \omega^{k}}\left(a \omega_{s}\right) \frac{d^{k} A_{s}(b)}{d b^{k}}\right]
$$

Then, since $\hat{g}^{\prime}\left(\omega_{0}\right)=0$, setting:

$$
a_{s}=\frac{\omega_{0}}{\omega_{s}}
$$

one has that:

$$
T_{s l}\left(b, a_{s}\right) \approx A_{s}(b) e^{i \omega_{s} b}
$$

up to a second order error.

Remark: Notice that such a simple calculus is not the consequence of the stationary phase estimations described in sections IV and V, which in such a particular case fail to yield approximate expressions for the wavelet transform (since we use the Morlet wavelet, the stationary points of the argument of the integrand are infinite order stationary points). Nevertheless, the philosophy of the technique is the same.

One can then use the same technique than the one used for ridge extraction to determine the value of $\omega_{s}$ : compute the wavelet transform of $s l(t)$ for a fixed value $a_{0}$ of the dilation parameter, and the derivative of its phase with respect to the translation parameter $b$ : the resulting frequency $\omega_{1}$ will yield a corresponding value $a_{1}=\frac{\omega_{0}}{\omega_{1}}$; then compute $T\left(b, a_{1}\right)$ and iterate the algorithm. The fixed point of the iteration will yield an estimate for $\omega_{s}$, the error being of second order in the derivatives of $A_{s}$.

\section{The case of two spectral lines:}

Assume now that $s(t)$ is the sum of two spectral lines, and that the corresponding amplitudes are regular and slowly varying enough so that the preceding approximations are still valid. Clearly, if the two frequencies are sufficiently far away from each other, the fast decay of $\hat{g}$ will allow to treat them independently, and to separate them. If not, 
one still can choose a sufficiently large $\omega_{0}$ to enforce the resolution of the wavelets in the Fourier space; but as stressed in section VI-2, such a trick is not so convenient for numerical computations, since it involves convolutions of the signal by wavelets with a large support. Moreover, increasing the central frequency of the analysing wavelet increase in the same time the values of the derivatives of $\hat{g}$ appearing in eq. $(V I-20)$, so that the asymptotic assumptions are no longer valid. It is actually possible to refine the method, by cancelling the interferences between the wavelet transforms of the two components. Consider for simplicity the case where the amplitudes of the two components are constant:

$$
s(t)=A_{1} \cos \left(\omega_{1} t\right)+A_{2} \cos \left(\omega_{2} t+\delta_{2}\right)
$$

Then:

$$
T(b, a)=A_{1} \overline{\hat{g}}\left(a \omega_{1}\right) e^{i \omega_{1} b}\left[1+C(a) e^{i\left(\omega_{2}-\omega_{1}\right) b+i \delta_{2}}\right]
$$

where

$$
C(a)=\frac{A_{2} \overline{\hat{g}}\left(a \omega_{2}\right)}{A_{1} \overline{\hat{g}}\left(a \omega_{1}\right)}
$$

Let $\varepsilon<\left|\frac{2 \pi}{\omega_{2}-\omega_{1}}\right|$ be a non-negative real number, and let:

$$
T=n\left|\frac{2 \pi}{\omega_{2}-\omega_{1}}\right|+\varepsilon
$$

for some positive integral number $n$. Introduce:

$$
\Omega_{a}=\frac{1}{T} \int_{b_{0}}^{b_{0}+T} \frac{\partial \Psi(b, a)}{\partial b} d b
$$

A straightforward computation shows that if $C(a)<1, \Omega_{a}=\omega_{1}+O\left(\frac{\varepsilon}{n}\right)$, and if $C(a)>1$, then $\Omega_{a}=\omega_{2}+O\left(\frac{\varepsilon}{n}\right)$. Obviously, the value of $C(a)$ depends only on the value of the dilation parameter one started with. Starting from $a_{0}$, and setting $a_{1}=\frac{\omega_{0}}{\Omega_{a}}$, a simple analysis (see e.g. [Se1]) shows that $\Omega_{a_{1}}$ is a fixed point of $\Omega$. One then has an iterative method to estimate the frequencies of the spectral lines. Notice that in that case, the error is proportional to $\frac{\varepsilon}{n}$, and that the signal being assumed asymptotic, $n$ can be made large enough to allow to neglect the error. A more detailed analysis of the convergence properties of the algorithm in the case of two spectral lines can be found in $[\mathrm{Gu}]$.

\section{The general algorithm:}

The spectral lines detection algorithm is the straightforward generalisation of the above discussion. Let $s(t)$ be the input signal, containing a certain number of spectral lines $\omega_{1}, \ldots \omega_{N}$. Let $a_{0}$ be a starting dilation parameter, and $T$ a (large) real number. Set:

$$
a_{i+1}=\frac{\omega_{0}}{\Omega_{a_{i}}}
$$


Then the $\left\{a_{i}\right\}$ sequence converges numerically very rapidly to a fixed point $a_{\infty}$, such that:

$$
a_{\infty} \approx \frac{\omega_{0}}{\omega_{k}}
$$

for some $k=1, \ldots N$. Moreover, different starting values of the dilation parameter $a_{0}$ will yield different spectral lines.

It must also be remarked that once a spectral line has been detected, it can be extracted by means of the factorisation property: indeed, consider for simplicity the case of two spectral lines. Then:

$$
\frac{1}{\overline{\hat{g}}\left(\omega_{0}\right)} T\left(b, \frac{\omega_{0}}{\omega_{1}}\right) \approx A_{1}(b) e^{i \omega_{1} b}+K(b) e^{i \omega_{2} b}
$$

where:

$$
K(b)=A_{2}(b) \frac{\overline{\hat{g}}\left(\frac{\omega_{0} \omega_{2}}{\omega_{1}}\right)}{\overline{\hat{g}}\left(\omega_{0}\right)}<A_{2}(b)
$$

so that the relative amplitude of the second component has decreased. One can iterate such a procedure by considering the left hand side of eq. $(V I-30)$ as a new input signal, and then neglect the second component after a certain number of iterations. In such a case, the first component is completely characterised, and can eventually be removed from the $s(t)$ signal. Examples of such spectral lines substractions will be shown in the next section.

\section{Separation of the contribution of each spectral line:}

In the case of the two frequencies signal, we saw that it is possible to exactly determine the values of the frequencies. Unfortunately, the modulus of the restriction of the wavelet transform to the values of the dilation parameter corresponding to those frequencies are oscillating functions. Nevertheless, for a fixed number of spectral lines of frequencies $\omega_{n}, n=1, . . N$, it is possible to 'disentangle' the components and then determine the amplitude modulation law of each component independently, without changing the time and frequency supports of the wavelets. For this purpose, one may assume that $N$ and the $\omega_{n}$ have previously been determined (this is possible to do thanks to the spectral lines estimation algorithm and the factorisation property described before). Linear transformations on the restrictions of the wavelet transform to these values of the dilation parameter lead to a linear system, whose solution yields the amplitude modulation law of all spectral lines (or more precisely the first order term in their asymptotic development).

\section{Numerical results:}

The first application of wavelet based spectral lines estimations was an application to NMR spectroscopy, where a spectral line corresponds to an excitation degree of freedom of a molecule. One then has to determine exactly the value of the frequency each spectral lines, and to separate the spectral lines of the studied molecules from those of the surrounding 
medium. This is what is exhibited in figs. VI-9. Fig. VI-9-a represents the Fourier spectrum of the original NMR signal, where the most important spectral line corresponds to the sorrounding medium, and where one is interested in the extraction and separation of the other ones, which are negligible in amplitude. Fig. VI-9-b represents the Fourier spectrum of the same signal, after the removal of the most important spectral line. Spectral lines estimations are also useful for noise filtering. Fig. VI-10-a represents the Fourier spectrum of a sum of spectral lines imbedded in noise, and fig. VI-10-b shows the sum of the extracted spectral lines. Let us finally show an example where the algorithms are used for modelisation in the aim of resynthesis. Figs. VI-11 show the extraction of 3 harmonics of the 16 main harmonics of the syllabe ' $l a^{\prime}$. The resynthesis is obtained by summing up all the harmonics [Gu]. 


\section{VII: APPLICATIONS TO MATCHED FILTERING:}

As stressed in section II, the computation of the wavelet (resp. Gabor) coefficients $T(b, a)$ (resp. $G(b, \omega)$ ) of $s(t)$ can be thought of as band-pass filtering of the signal, which localize it into cells (in the time-frequency plane) of constant relative bandwidth $\frac{\Delta \nu}{\nu}$ (resp. constant absolute bandwidth $\Delta \nu$ ). It is then natural to combine such filters to build by hand other filters, adapted to special kinds of signals. This is the purpose of matched filtering, the matched filter being defined as the linear filter which optimizes the signal to noise ratio of the output (see e.g. [Pap], [Bl]). Notice that in such an approach, it is also natural to use the simplified reconstruction formula $(I I-7)$ to recover the signal as the sum over all the filtered components.

We consider here the case of asymptotic locally monochromatic signals (for which approximate expressions for the wavelet and Gabor transforms are available), and we restrict to the special case of wavelet analysis; analogous algorithms can be derived in the framework of Gabor analysis.

We will describe two algorithms, called the ridge coincidence algorithm and the ridge rectification algorithm respectively, whose purpose is detection and time-location of a-priori well known asymptotic locally monochromatic components $s(t)$ in a signal, imbedded in an input signal $\tilde{s}(t)$. These two algorithms model filters close to the matched filter, in the sense that the output signal exhibits a sharp peak at the estimated date, like the autocorrelation function of $s(t)$.

Both algorithms basically follow the same philosophy. They first use the wavelets to localise the signal into time-frequency cells of constant relative bandwidth, and the contributions of such cells are summed in different ways. Finally, the same operations are performed with time-shifted copies of the cells. The output signal is in both cases different than the autocorrelation function of $s(t)$, but basically has the same characteristics.

Let us stress that one can build in the same way many slightly different versions of such algorithms; our goal in this section is not to present algorithms in a final form, but rather to illustrate how wavelet analysis can be used to get approximations of the matched filter. Let us also point out the simple geometrical interpretation of these algorithms in the time-frequency half-plane.

\section{VII-1: The Ridge Coincidence Algorithm:}

Let $s \in L^{2}(\mathbf{R})$ be an asymptotic locally monochromatic signal of the form given by eq. $(I I I-8)$, and let $g \in H^{2}(\mathbf{R})$ be an analytic analysing wavelet, written in the form given by eq. $(I V-3)$. Denote by:

$$
\mathcal{R}=\left\{(b, a) \in \Omega ; a=a_{r}(b)\right\}
$$

the ridge of the wavelet transform $T(b, a)$ of $s(t)$. It is convenient to introduce here the $\tau$-shifted skeleton operator $\mathcal{F}_{\tau}^{\mathcal{R}}: L^{2}(\mathbf{R}) \rightarrow L^{2}(\mathbf{R}):$ if $\tilde{s} \in L^{2}(\mathbf{R})$, and $T_{\tilde{s}}$ is its wavelet 
transform:

$$
\left[\mathcal{F}_{\tau}^{\mathcal{R}} . \tilde{s}\right](t)=\sqrt{\frac{2}{\pi}} \frac{\sqrt{a_{r}(t)^{2}\left|\Phi_{\left(t, a_{r}(t)\right)}^{\prime \prime}(t)\right|}}{\overline{g(0)}} e^{-i \frac{\pi}{4} \operatorname{Sgn}\left[\Phi_{\left(t, a_{r}(t)\right)}^{\prime \prime}(t)\right]} T_{\tilde{s}}\left(t+\tau, a_{r}(t)\right) \quad(V I I-2)
$$

which is the $\tau$-shifted analogue of the skeleton operator introduced in eq. $(I V-17)$. At $\tau=0, \mathcal{F}_{\tau}^{\mathcal{R}}$ associates with $\tilde{s}$ its wavelet transform restricted to the $\mathcal{R}$ ridge, and corrected by a (local) normalisation function and phase shift, needed to compensate the terms introduced by the stationary phase evaluation of the integrals. For $\tau \neq 0$, the wavelet transform is now restricted to a $\tau$-shifted copy of the $\mathcal{R}$ ridge:

$$
\mathcal{R}^{\tau}=\left\{(b, a) \in \Omega ; a=a_{r}(b-\tau)\right\}
$$

Clearly, when $\tilde{s}(t)=s\left(t-\tau_{0}\right), \mathcal{R}^{\tau}$ coincides with the ridge of $\tilde{s}$, so that both functions:

$$
\begin{gathered}
\mathcal{S C}(\tau)=<\mathcal{F}_{\tau}^{\mathcal{R}} \cdot \tilde{s}, Z_{s}> \\
\mathcal{S E}(\tau)=\left\|\mathcal{F}_{\tau}^{\mathcal{R}} \cdot \tilde{s}\right\|^{2}
\end{gathered}
$$

are maximal at $\tau=\tau_{0}$ (to see this, just take for $\mathcal{F}_{\tau}^{\mathcal{R}} . \tilde{s}$ its expression provided by the stationary phase argument in section IV, and evaluate the scalar products). $\mathcal{S C}$ is called the skeleton correlation function, and $\mathcal{S E}$ is the skeleton energy function. They are to be compared with the autocorrelation function $\mathcal{C}$, output of the matched receiver:

$$
\mathcal{C}(\tau)=\int Z_{s}(t) \overline{Z_{s}(t-\tau)} d t
$$

Consider now $\tilde{s}(t)$ of the form:

$$
\tilde{s}(t)=\sum_{i=1}^{N} A_{i} s\left(t-\tau_{i}\right)+r(t)
$$

for some real numbers $A_{i}$ and $\tau_{i}$, and some deterministic or random $r(t) . \tilde{s}(t)$ contains $N$ shifted copies of $s(t)$, and the corresponding ridge is a multicomponent ridge, which contains in particular $\mathrm{N}$ copies of the ridge of $s(t)$. Assume moreover that the $\tau_{i}$ parameters are large enough to ensure that these components of the ridge are non-interacting, in the sense defined in section VI. Then $\mathcal{S C}(\tau)$ is still locally maximal (more precisely it exhibits sharp peaks) at $\tau=\tau_{i}, i=1, . . N$, at least as soon as the wavelet coefficients $T_{r}\left(b+\tau, a_{r}(b)\right)$ are small compared with all the $A_{i} T_{s}\left(b, a_{r}(b)\right)$. This in a first step allows the determination of the time delay parameters $\tau_{i}$, and in a second step the determination of the $A_{i}$ parameters, by using the factorisation property described in section VI. In the opposite case, additional local maxima appear; one says that the false alarm probability becomes more important.

Note that the local maxima of $\mathcal{S C}(\tau)$ correspond to the cases where the ridges of $\tilde{s}(t)$ coincide with the theoretical ridge of $s(t)$. This explains the name given to the algorithm. 


\section{Numerical results:}

As an example, consider an hyperbolically frequency modulated signal, starting at $\tau_{0}=0$. Figs. VII-1-a to VII-1-c represent respectively the $\mathcal{C}(\tau), \mathcal{S E}(\tau)$ and $\mathcal{S C}(\tau)$ functions for such a signal. Notice that $\mathcal{S C}(\tau)$ is sharply peaked at $\tau=0$. In the case of a noisy signal, up to some critical value of the signal to noise ratio, time estimation via the skeleton correlation is still possible, as exhibited in Figs. VII-2-a to VII-2-c (here the asymptotic signal is the same than previously, and the noise is a uniform random signal, whose amplitude equals 10 times the maximal amplitude $s(t))$. The performances are then comparable to those of the matched filter.

\section{VII-2: The Ridge Rectification Algorithm:}

We now describe another algorithm dedicated to matched filtering. The main idea of this algorithm is to transform a signal with a given ridge into another signal, with a ridge as close as possible to a vertical ridge:

$$
\mathcal{R}_{b_{0}}=\left\{(b, a) \in \Omega ; b=b_{0}\right\}
$$

for some fixed $b_{0}$. Then, use the simplified reconstruction formula to construct the output of the algorithm from the modified wavelet coefficients. We describe here the generic structure of the algorithm in the particular case of hyperbolic frequency modulation laws, for which it is known that representations associated with the affine group are optimal in terms of energy localisation in the time-scale plane (see e.g. [Be-Be]).

The ridge rectification algorithm goes as follows:

In a first step, pick a fixed number of dilation parameters $a_{i}, i=1, . . N$, chosen in such a way that the corresponding relative bandwidth

$$
\frac{\Delta a}{a}=2 \frac{a_{i}-a_{i-1}}{a_{i}+a_{i-1}}
$$

is constant. To these dilation parameters are then associated the proper frequencies of the corresponding dilated wavelets:

$$
\nu_{i}=\frac{\phi_{g}^{\prime}(0)}{2 \pi a_{i}}
$$

and then the time delays $t_{i}$ such that:

$$
\nu_{i}=\frac{1}{2 \pi} \phi_{s}^{\prime}\left(t_{i}\right)
$$

(see fig. VII-3-a). Fix a reference time $T$, and set:

$$
\theta_{i}=T-t_{i}
$$

In a second step, compute the wavelet transform of the input signal for these fixed values of the dilation parameter, and time-translate the i-voice by a time-delay equal to 
$\theta_{i}$. One then gets modified wavelet coefficients, which no longer satisfy the reproducing kernel equation. This in particular means that one does not have any control a priori on the signal reconstructed from these modified coefficients. Nevertheless, a ridge extraction gives a vertical ridge like in eq. (VII-9). Another modification is then necessary.

In a third step, to achieve coherence between all the voices, the output of each voice is multiplied by a corrective function:

$$
\operatorname{Corr}_{k}(t)=\sqrt{\frac{2}{\pi}} \frac{\sqrt{a_{k}^{2} \Phi_{\left(t-\theta_{k}, a_{k}\right)}^{\prime \prime}\left(t_{s}\right)}}{\overline{g(0)}}
$$

and a phase shift:

$$
\delta \phi_{k}=-2 \pi \phi_{s}\left(t_{s}\left(t-\theta_{k}, a_{k}\right)\right)+C s t
$$

which are completely determined by the a-priori known ridge.

Finally, the modified outputs of all voices are summed up in a coherent way:

$$
W(t)=\sum_{i=1}^{N} \operatorname{Corr}_{k}(t) e^{-\delta \phi_{k}} T_{s}\left(t-\theta_{k}, a_{k}\right)
$$

or in a partially coherent way:

$$
E(t)=\sum_{i=1}^{N}\left|\operatorname{Corr}_{k}(t) T_{s}\left(t-\theta_{k}, a_{k}\right)\right|^{2}
$$

The generic structure of the algorithm is summarized in figs. VII-3-a and VII-3-b..

It is then not difficult to show that if the receiver is matched to the frequency modulation law of the incoming signal, i.e. if the incoming signal is hyperbolically frequency modulated, both $W(t)$ and $E(t)$ are maximal at $t=T$. If the input signal is now of the form given by eq. (VII-7), the output signal will exhibit sharp peaks at all the $t=T+\tau_{i}$, yielding precise estimates for the $\tau_{i}$ 's.

\section{Implementation and numerical results:}

The main point in the practical implementation of the algorithm is the choice of the analysing wavelet, and the determination of the number of different dilation parameters to be taken into account. For a fixed analysing wavelet, the number of voices is determined by the constant relative bandwidth constraint, and by the specification of the constant overlap of the corresponding dilated wavelets in the Fourier space: the Fourier transforms of the dilated wavelets intersect when they take a fixed value, set to $\epsilon$ times the maximal value $\hat{g}\left(\omega_{0}\right)$, for some fixed $\epsilon<1$.

Let us consider the simple example of the hyperbolically frequency modulated signal, with instantaneous frequency:

$$
\nu(t)=\frac{\nu_{s}}{1+\alpha t} \quad t \in[0, T]
$$


and set $\nu_{e}=\nu(T)$. The values of the considered dilation parameters $a_{k}$ are then completely determined (see [E-To] for more details):

$$
a_{k}=\frac{\nu_{0}}{\nu_{s}} \frac{\left(1+\frac{1}{2 Q_{\epsilon}}\right)^{k+1}}{\left(1-\frac{1}{2 Q_{\epsilon}}\right)^{k-1}}
$$

Here $Q_{\epsilon}=\omega_{0} \sqrt{-2 \ln (\epsilon)}$ is a surtension factor.

Figs. VII-4-a and VII-4-b represent respectively $E(t)$ and $|W(t)|^{2}$ in the case $N=12$ and $\omega_{0}=15$, exhibiting sharp maxima at $t=T$. In the presence of a Gaussian noise (Figs. VII-5-a and VII-5-b), the signal to noise ratio at the output $W(t)$ enables an accurate time estimation, and may be considered as an unbiased time estimator. Various attempts [E-To] indicate that the improvement of signal to noise ratio due to the coherent receiver is near $B T, B$ being the bandwidth of the received signal.

\section{Remark: ridge rectification and bat's sonar receiver:}

Such results may appear as common ones for signal processing. Nevertheless, the model described here is in a strong connexion with biological data about bats. Bats use an ultrasonic sonar system to detect obstacles during cruise, to locate and pursuit their preys [Si]. Among various species of bats, Vespertillionids emit wideband signals, the amplitude and the frequency of which are modulated in such a way that they can be considered as asymptotic (see fig. VI-2-a). The acoustic receiver of bats is built up with nervous cells, the response of which is similar to that of tuned circuits. The relative bandwidth of these cells is constant, like that of wavelets. These cells may then be represented as a "wavelet receiver", and wavelet representation is then well suited to model such a receiver. Recent results by J. Simmons [Si] have moreover pointed out two main facts, which enforce the connexion with wavelet representation:

-Auditory representations of echoes is displayed by nervous cells as a spectrogram (or scalogram) due to the Corti's cells acting as constant relative bandwidth filters.

-The outputs of such tuned cells are the processed parts of the incoming echoe relative to the relative bandwidth of the filters. These outputs (nervous response) are called neurograms, and appear to be deeply related to scalograms. 


\section{VIII: CONCLUSIONS:}

We have reviewed here the main properties of wavelet and Gabor representations when applied to asymptotic signals or composite signals of asymptotic type. The main ingredient of the applications we described is that time-frequency representations allow to separate components of signals located in different regions of the time-frequency plane, and then to synthetise them independently.

It is then natural to see that the techniques described above give remarkable results in the case of asymptotic signals, and in the case of composite signals of asymptotic type when the components do not interact in the time-frequency plane; nevertheless, it is still difficult to isolate components that strongly interact in the time-frequency plane, as for instance in the case of the bubbles described in section VI. In such cases, new techniques are needed, and there is some hope that wavelet packets [Co-Me-Qu-Wi],[To3] or even more general decompositions and adaptive analysis schemes could yield better results.

Another point that has to be stressed is that in the analysis described here, which is essentially concerned with a careful study of the phase of the time-frequency representation, there is absolutely no difference between wavelet and Gabor analysis (of course the explicit expressions and the algorithms are slightly different, but the general procedure is completely identical). The only difference that appears between the two approaches lies in the behaviour of the squared modulus of the representation, i.e. the time-frequency energy density. This is the consequence of a well known property of time-frequency representations, that is that a given representation is optimal (in the sense of energy localisation) for only one type of frequency modulation law.

Finally, notice that the computations of sections IV and V can trivially be generalised to the two-dimensional wavelet transform with rotations, described un $[\mathrm{Mu}]$. The only difference is that the notion of wavelet curve has to be replaced by that of wavelet surface. 


\section{APPENDIX: THE STATIONARY PHASE ARGUMENT:}

Let us describe briefly here the approximation techniques we use in this paper. We derive in a simple way the first terms of the asymptotic developments of $T(b, a)$ we start from in sections IV and V (the approximations for $G(b, \omega)$ and $T^{\lambda}(b, a)$ go exactly in the same way).

From eq. $(I V-4)$, one has the following expression for the wavelet transform of $s \in L^{2}(\mathbf{R}):$

$$
T(b, a)=\frac{1}{2 a} \int_{-\infty}^{\infty} M_{(b, a)}(t) e^{i \Phi_{(b, a)}(t)} d t
$$

where:

$$
M_{(b, a)}(t)=A_{s}(t) A_{g}\left(\frac{t-b}{a}\right)
$$

and

$$
\Phi_{(b, a)}(t)=\phi_{s}(t)-\phi_{g}\left(\frac{t-b}{a}\right)
$$

From now on, we will assume that both $g(t)$ and $s(t)$ are analytic functions, so that their series development make sense.

\section{A-1: $g(t)$ and $s(t)$ asymptotic:}

Assume that both $s(t)$ and $g(t)$ are asymptotic signals in the sense defined in section III. Then $T(b, a)$ is defined by a rapidly oscillating integral, so that asymptotic techniques can be used (see e.g. [Co]). Moreover, because of the regularity and the fast decay of $g(t)$, the integration bounds do not contribute to the asymptotic development of $T(b, a)$. Let $t_{s}=t_{s}(b, a)$ be such that:

$$
\Phi_{(b, a)}^{\prime}\left(t_{s}\right)=0
$$

Then, by general arguments, the essential contribution to $(A-1)$ is provided by the neighborhood of such points. Moreover, assuming for simplicity that $t_{s}$ is the unique point such that $(A-4)$ holds and that $M_{(b, a)}$ is non negligible, then the first term in the asymptotic development of $T(b, a)$ reads:

$$
T_{(0)}(b, a)=\frac{M_{(b, a)}\left(t_{s}\right) e^{i \Phi_{(b, a)}\left(t_{s}\right)}}{2 a} \int_{-\infty}^{\infty} e^{i \frac{1}{2}\left(t-t_{s}\right)^{2} \Phi_{(b, a)}^{\prime \prime}\left(t_{s}\right)} d t
$$

A straightforward Gaussian integration thus yields:

$$
T_{(0)}(b, a)=\sqrt{\frac{\pi}{2}} \frac{Z_{s}\left(t_{s}\right) \overline{\frac{1}{a} g\left(\frac{t_{s}-b}{a}\right)}}{\sqrt{\left|\Phi_{(b, a)}^{\prime \prime}\left(t_{s}\right)\right|}} e^{i \frac{\pi}{4} \operatorname{Sgn}\left[\Phi_{(b, a)}^{\prime \prime}\left(t_{s}\right)\right]}
$$

where we have assumed that:

$$
\Phi_{(b, a)}^{\prime \prime}\left(t_{s}\right) \neq 0
$$


The second term of the asymptotic development, i.e. the term proportional to the first derivative of $M_{(b, a)}$ is easy to estimate too:

$$
T_{(1)}(b, a)=\frac{M_{(b, a)}^{\prime}\left(t_{s}\right) e^{i \Phi_{(b, a)}\left(t_{s}\right)}}{2 a} \int_{-\infty}^{\infty}\left(t-t_{s}\right) e^{i \frac{1}{2}\left(t-t_{s}\right)^{2} \Phi_{(b, a)}^{\prime \prime}\left(t_{s}\right)} d t=0
$$

which proves the strongness of the approximation.

\section{A-2: $s(t)$ asymptotic and $|g(t)|$ Gaussian :}

In the particular case of an analysing wavelet with a Gaussian amplitude:

$$
g(t)=e^{-\frac{t^{2}}{2}} e^{i \phi_{g}(t)}
$$

the asymptotic assumption on $g(t)$ can be dropped as follows: instead of considering the Taylor series development of $M_{(b, a)}$, consider the development of $A_{s}(t)$ only. This also means that one assumes the variations of $A_{s}$ to be slow compared with those of the Gaussian amplitude of the wavelets. The first term in the asymptotic development of $T(b, a)$ then reads:

$$
T_{(0)}(b, a)=\frac{1}{2 a} A_{s}\left(t_{s}\right) e^{i \Phi_{(b, a)}\left(t_{s}\right)} \int_{-\infty}^{\infty} e^{-\frac{1}{2}\left(\frac{t-b}{a}\right)^{2}} e^{i \frac{1}{2}\left(t-t_{s}\right)^{2} \Phi_{(b, a)}^{\prime \prime}\left(t_{s}\right)} d t
$$

which then once more reduces to a Gaussian integration, and yields:

$$
\begin{aligned}
& T_{(0)}(b, a)=\left|T_{(0)}(b, a)\right| e^{i \Psi_{(0)}(b, a)} \\
& (A-12-a) \\
& \left|T_{(0)}(b, a)\right|=\sqrt{\frac{\pi}{2}} \frac{A_{s}\left(t_{s}\right) e^{-\frac{1}{2} \frac{\left(t_{s}-b\right)^{2} \Phi_{(b, a)}^{\prime \prime}\left(t_{s}\right)^{2}}{\Phi_{(b, a)}^{\prime \prime}\left(t_{s}\right)^{2}+\frac{1}{a^{4}}}}}{\left[1+a^{4} \Phi_{(b, a)}^{\prime \prime}\left(t_{s}\right)^{2}\right]^{\frac{1}{4}}} \\
& (A-12-b) \\
& \Psi_{(0)}(b, a)=\phi_{s}\left(t_{s}\right)-\phi_{g}\left(\frac{t_{s}-b}{a}\right)+\frac{1}{2} \frac{\left(t_{s}-b\right)^{2} \Phi_{(b, a)}^{\prime \prime}\left(t_{s}\right)}{1+a^{4} \Phi_{(b, a)}^{\prime \prime}\left(t_{s}\right)^{2}}+\frac{1}{2} \arctan \left[a^{2} \Phi_{(b, a)}^{\prime \prime}\left(t_{s}\right)\right] \\
& (A-12-c)
\end{aligned}
$$

It must be noticed here that the next term in the asymptotic development, proportional to the derivative of $A_{s}(t)$ is not identically zero in that case. Its computation still reduces to a Gaussian integration, and yields:

$$
\begin{aligned}
& T_{(1)}(b, a)=\left|T_{(1)}(b, a)\right| e^{i \Psi_{(1)}(b, a)} \quad(A-13-a) \\
& \left|T_{(1)}(b, a)\right|=\sqrt{\frac{\pi}{2}} \frac{A_{s}^{\prime}\left(t_{s}\right) e^{-\frac{1}{2} \frac{\left(t_{s}-b\right)^{2} \Phi_{(b, a)}^{\prime \prime}\left(t_{s}\right)^{2}}{\Phi_{(b, a)}^{\prime \prime}\left(t_{s}\right)^{2}+\frac{1}{a^{4}}}}}{\left[1+a^{4} \Phi_{(b, a)}^{\prime \prime}\left(t_{s}\right)^{2}\right]^{\frac{3}{4}}}\left|t_{s}-b\right| \quad(A-13-b) \\
& \Psi_{(1)}(b, a)=\phi_{s}\left(t_{s}\right)-\phi_{g}\left(\frac{t_{s}-b}{a}\right)+\frac{1}{2} \frac{\left(t_{s}-b\right)^{2} \Phi_{(b, a)}^{\prime \prime}\left(t_{s}\right)}{1+a^{4} \Phi_{(b, a)}^{\prime \prime}\left(t_{s}\right)^{2}}+\frac{3}{2} \arctan \left[a^{2} \Phi_{(b, a)}^{\prime \prime}\left(t_{s}\right)\right] \\
& (A-13-c)
\end{aligned}
$$

Throughout this paper, the symbol $\approx$ will mean that one has replaced an expression by the first order term of its asymptotic development. 


\section{FIGURE CAPTIONS:}

Fig. VI-1-a: Constant amplitude hyperbolically modulated signal: Phase and modulus of the wavelet transform. The ridge of the transform is drawed on the phase plot.

Fig. VI-1-b: Constant amplitude hyperbolically modulated signal: Modulus and frequency modulation laws, extracted from the wavelet transform.

Fig. VI-2-a: Detection or cruise sonar signal emitted by a Vespertillionid bat (Myotis mystacinus): Ridges and modulus of the wavelet transform.

Fig. VI-2-b: Sonar signal emitted by a Vespertillionid bat, with additional uniform noise: Modulus and frequency modulation laws, extracted from the wavelet transform.

Fig. VI-3: Sonar signal emitted by a Vespertillionid bat, with additional uniform noise: Modulus and frequency modulation laws, extracted from the phase of the associated analytic signal.

Fig. VI-4: Sum of two linearly frequency modulated signals, with close instantaneous frequencies: Phase and modulus of the wavelet transform with respect to a Morlet wavelet, with a standard value of the central frequency of the wavelet. The ridge is represented on the phase plot.

Fig. VI-5: Sum of two linearly frequency modulated signals, with close instantaneous frequencies: Phase and modulus of the wavelet transform with respect to a Morlet wavelet, with a larger value of the central frequency of the wavelet. The ridge is represented on the phase plot.

Fig. VI-6-a: Phase and ridges of the Gabor transform of a trumpet sound signal.

Fig. VI-6-b: Phase and ridges of the Gabor transform of a female voice signal.

Fig. VI-7: Ridges of the Gabor transform of the sum of two close spectral lines.

Fig. VI-8-a: Ridges of the Gabor transform of a clarinet sound signal: first example.

Fig. VI-8-b: Ridges of the Gabor transform of a clarinet sound signal: second example.

Fig. VI-9-a: Fourier spectrum of a NMR spectroscopy signal

Fig. VI-9-b: Same than fig. VI-9-a, after the removal of the main spectral line.

Fig. VI-10-a: Sum of spectral lines imbedded in noise.

Fig. VI-10-b: Same than fig. VI-10-a, after the removal of the noise.

Fig. VI-11-a: Wavelet decomposition of the syllabe ' $l a^{\prime}$; sampling frequency: 22510 Hz. Harmonic number $1, \nu=274.5 \mathrm{~Hz}$.

Fig. VI-11-b: Wavelet decomposition of the syllabe ' $l a^{\prime}$; sampling frequency: 22510 Hz. Harmonic number 5, $\nu=1374 \mathrm{~Hz}$.

Fig. VI-11-c: Wavelet decomposition of the syllabe ' $l a$ '; sampling frequency: 22510 Hz. Harmonic number 10, $\nu=2749.2 \mathrm{~Hz}$.

Fig. VII-1-a: Autocorrelation of a triangularly amplitude modulated and hyperbolically frequency modulated signal.

Fig. VII-1-b: Skeleton energy of a triangularly amplitude modulated and hyperbolically frequency modulated signal.

Fig. VII-1-c: Skeleton correlation of a triangularly amplitude modulated and hyperbolically frequency modulated signal. 
Fig. VII-2-a: Same than Fig. VII-1-a, with an additional uniform noise with amplitude ten times larger than the maximal amplitude of the asymptotic signal.

Fig. VII-2-b: Same than Fig. VII-1-b, with an additional uniform noise with amplitude ten times larger than the maximal amplitude of the asymptotic signal.

Fig. VII-2-c: Same than Fig. VII-1-c, with an additional uniform noise with amplitude ten times larger than the maximal amplitude of the asymptotic signal.

Fig. VII-3-a: Definition of the time delays $\theta_{k}$.

Fig. VII-3-b: Generic structure of the ridge rectification algorithm.

Fig. VII-4-a: $E(t)$ function in the case of an hyperbolically frequency modulated signal, and a receiver with $N=12$.

Fig. VII-4-b: $|W(t)|^{2}$ function in the case of an hyperbolically frequency modulated signal, and a receiver with $N=12$.

Fig. VII-5-a: Same than Fig VII-4-a, with an additional centered Gaussian noise, with input signal to noise ratio equal to $1 / 5$.

Fig. VII-5-b: Same than Fig VII-4-b, with an additional centered Gaussian noise, with input signal to noise ratio equal to $1 / 5$. 


\section{REFERENCES:}

[Be-Be]: J. Bertrand, P. Bertrand, Classification of affine Wigner functions via an extended covariance principle, Proc. of the 17th international colloquium on group theoretical methods in physics, Ste Adèle, Y. Saint Aubin and L. Vinet Eds., World Scientific (1990), 380-383.

[Bl]: R.E. Blahut, Principles and practice of information theory, Addison-Wesley publishing company, AW series in electrical and computer engineering (1987).

[Co-Gr-Tc]: J.M. Combes, A. Grossmann, Ph. Tchamitchian Eds., Wavelets, Proceedings of the first conference, Marseille (1987), Springer.

[Cop]: E. Copson, Asymptotic expansions, Cambridge University Press (1965).

[Da1]: I. Daubechies, The wavelet transform, time-frequency localisation and signal analysis, IEEE Trans. Inf. Th. 36 (1990) pp.961-1005.

[Da2]: I. Daubechies, Orthonormal bases of compactly supported wavelets, Comm. Pure Appl. Math. 41 (1988),909-996.

[Da-Pa]: I. Daubechies, T. Paul, Time-frequency localisation operators, a geometric phase space approach II: the use of dilations, Inverse problems 4 (1988), 661.

[De]: N. Delprat, Extraction of frequency modulation laws in sound synthesis, to appear in $[\mathrm{Me} 4]$.

[De-Gu-KM]: N. Delprat, P. Guillemain, R. Kronland-Martinet, Parameters estimation for non linear resynthesis methods of a time-frequency analysis of natural sounds, Proc. of the ICMC, Glasgow (1990), pp. 88-90.

[Di]: R.B. Dingle, Asymptotic expansions, their derivation and interpretation, Academic Press (1973).

[E]: B. Escudié, Wavelet analysis of asymptotic signals: A temptative model for bat sonar receiver, to appear in [Me4].

[E-Gr-KM-To]: B. Escudié, A. Grossmann, R. Kronland-Martinet, B. Torrésani, Analyse en ondelettes de signaux asymptotiques: emploi de la phase stationnaire, proceedings of the GRETSI conference, Juan-les-Pins (1989).

[E-Ma]: B. Escudié, W. Martin, Modélisation des signaux sonar animaux par représentation canonique du signal analytique, Proceedings of the GRETSI conference, Nice (1985), 25-29.

[E-To]: B. Escudié, B. Torrésani, Wavelet representation and time-scaled matched receiver for asymptotic signals, Proceedings of the conference EUSIPCO V, Barcelona (1990), North Holland, 305-308.

[Ga]: D. Gabor, Theory of communication, J. Inst. Elec. Eng. 903 (1946), 429.

[Gr-KM-Mo]: A. Grossmann, R. Kronland-Martinet, J. Morlet, Reading and understanding the continuous wavelet transform, in [Co-Gr-Tc].

[Gr-Mo]: A. Grossmann, J. Morlet, Decomposition of Hardy functions into square integrable wavelets of constant shape, SIAM J. of Math. An. 15 (1984),723.

[Gr-Mo-Pa1]: A. Grossmann, J. Morlet, T. Paul, Transforms associated with square integrable group representations I, J. Math. Phys. 27 (1985),2473. 
[Gr-Mo-Pa2]: A. Grossmann, J. Morlet, T. Paul, Transforms associated with square integrable group representations II, Ann. Inst. H. Poincaré, 45 (1986) 293.

[Gu]: P. Guillemain, Analyse et modélisation de signaux acoustiques: application de la transformée en ondelettes, DEA, LMA-Preprint (1990) Marseille.

[Gu-KM]: P. Guillemain, R. Kronland-Martinet, Parameters estimation through wavelet transform for synthesis of audio sounds, Preprint for the 90th AES convention (1991), Marseille.

[Gu-KM-Ma]: P. Guillemain, R. Kronland-Martinet, B. Martens, Estimation of spectral lines with the help of the wavelet transform, applications in NMR spectroscopy, to appear in [Me4].

[Ho-Tc]: M. Holschneider, Ph. Tchamitchian, Pointwise analysis of Riemann-Weierstrass nowhere differentiable function, Preprint CPT, Marseille (1989), to appear at Invent. Math.

[Ja]: S. Jaffard, Exposants de Holder en des points donnés, et coefficients d'ondelettes, CRAS 308, série 1 (1989), 79.

[Ko-Ge-dG]: K. Kodera, R. Gendrin, C. de Gilledary, Analysis of time-varying signals with small BT values, IEEE Trans. ASSP 26 (1978), 64.

[Me1]: Y. Meyer, Ondelettes et opérateurs: I Ondelettes, (1989), Hermann.

[Me2]: Y. Meyer, Ondelettes et opérateurs II Opérateurs de Calderon-Zygmund, (1990), Hermann.

[Me3]: Y. Meyer, Ondelettes et opérateurs III, to appear, Hermann.

[Me4]: Y. Meyer Ed., Wavelets and applications, Proceedings of the second wavelet conference, Marseille (1989), Masson, to appear.

[Mu]: R. Murenzi, Ondelettes multidimensionnelles et applications a l'analyse d'images, PhD Thesis, IPT Louvain (1990).

[Pap]: A. Papoulis, Probability, random variables and stochastic processes, McGraw-Hill series in electrical engineering, 2nd edition (1989).

[Pi-Ma]: B. Picinbono, W. Martin, Représentation des signaux par amplitude et phase instantanées, Annales des Télécommunications 38 (1983), 179-190.

[Se1]: K. Seip, Some remarks on a method for detecting spectral lines in a signal, Preprint CPT-89/P.2252, Marseille.

[Se2]: K. Seip, Curves of maximal modulus in coherent state representations, Preprint CPT-89/P.2254, Marseille.

[Si]: J. Simmons, Clutter interference and the integration time of echoes in the echolocating bat: Eptesicus Fuscus, JASA 86 (1989),1318.

[Tc-To]: Ph. Tchamitchian, B. Torrésani, Ridge and Skeleton extraction from wavelet transform, Preprint CPT-90/P.2467, Marseille, to appear in "Wavelets and their Applications", Coifman \& Al Eds, Jones\&Bartlett, Boston (1991).

[To1]: B. Torrésani, Wavelet analysis of asymptotic signals: Ridge and Skeleton of the transform, to appear in [Me4].

[To2]: B. Torrésani, Wavelets associated with representations of the affine Weyl-Heisenberg group, Preprint CPT-90/P.2390, Marseille, to appear at J. Math. Phys. (June 1991).

[To3]: B. Torrésani, Time-Frequency Representations: wavelet packets and optimal decomposition, Preprint CPT-90/P.2466, Marseille, to appear at Ann. Inst. H. Poincaré. 


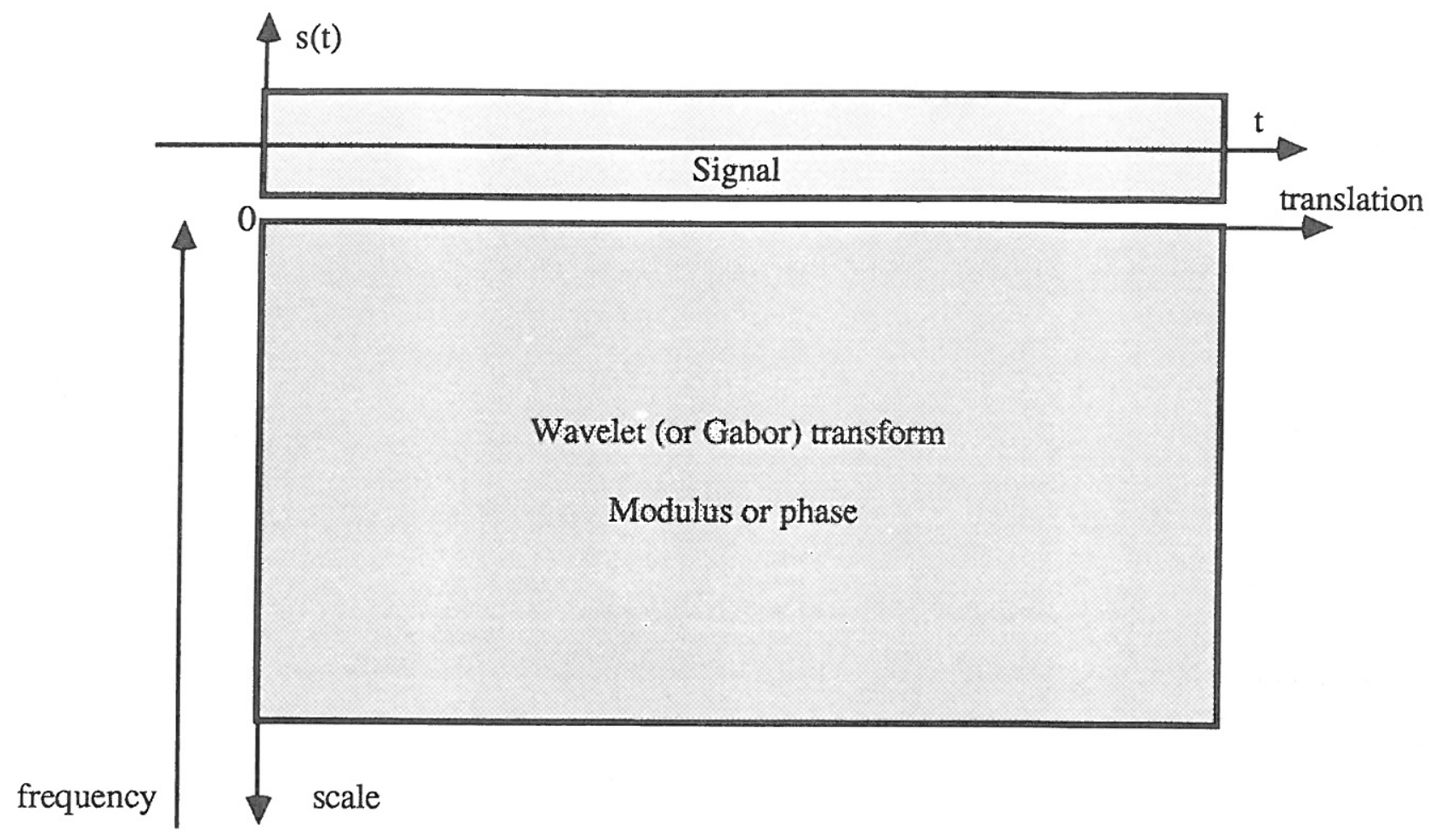

Figure VI-0 


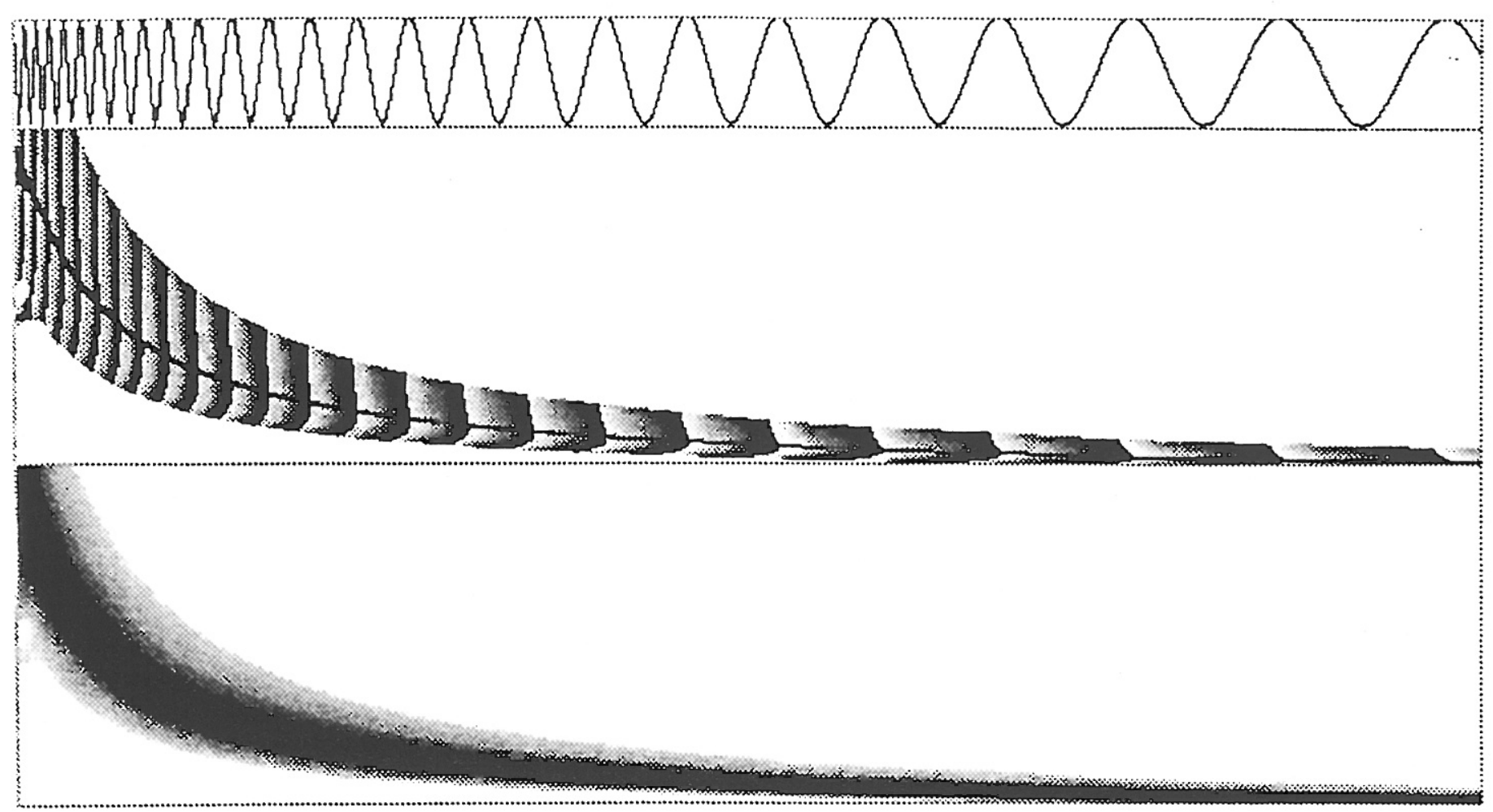

FIG. VI-I-a 


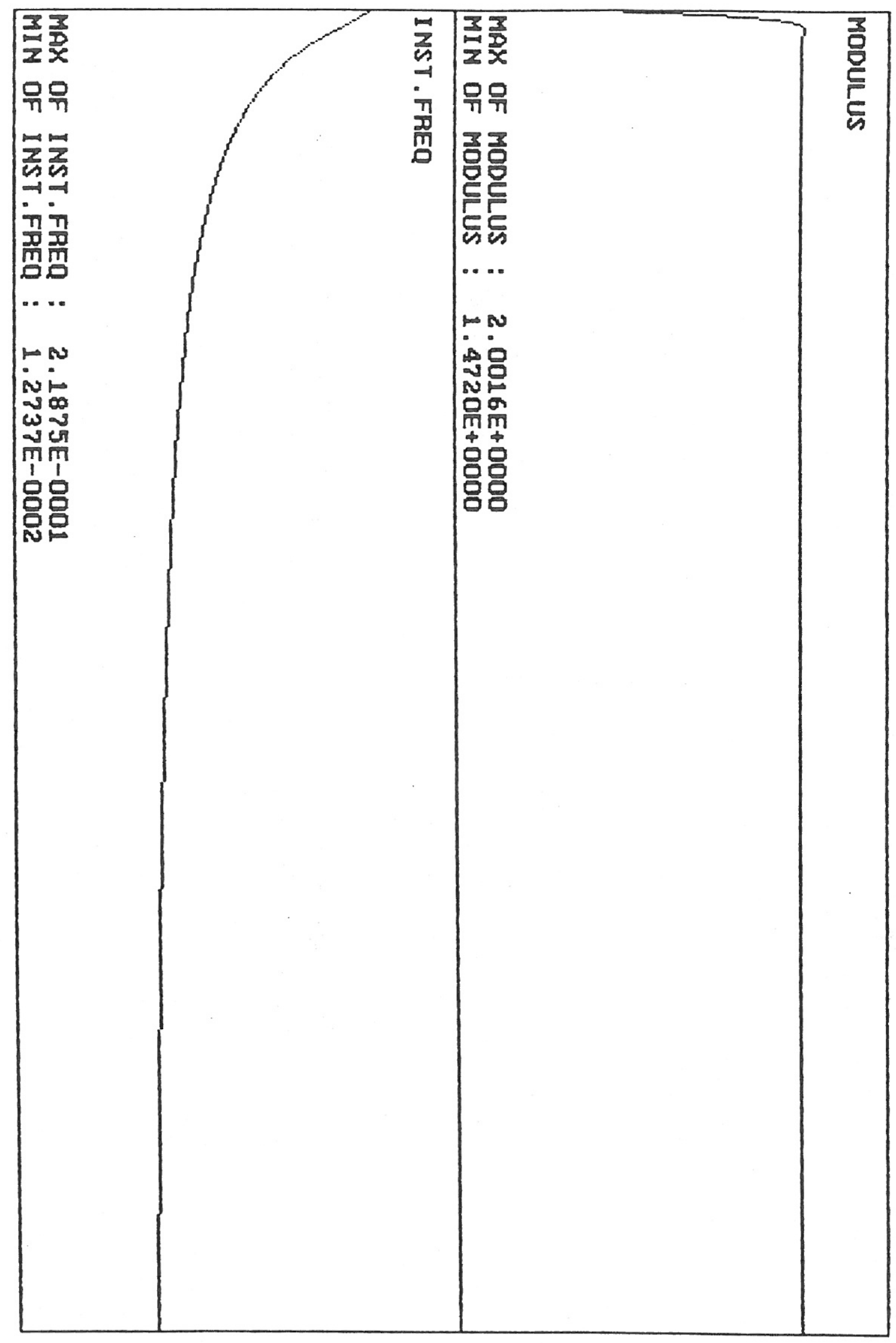

FIG. VI-1-b 


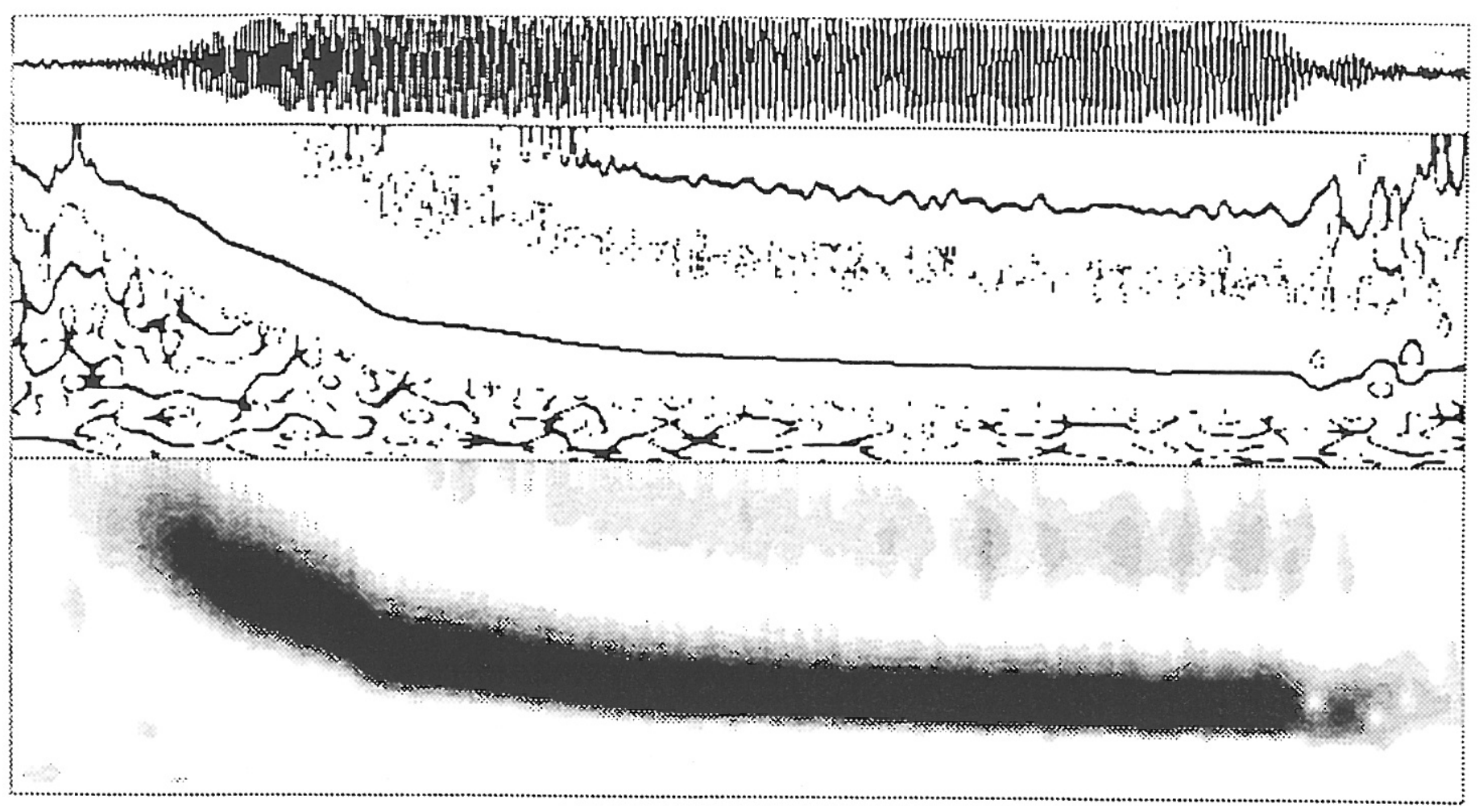

FIG. VI-2-a 


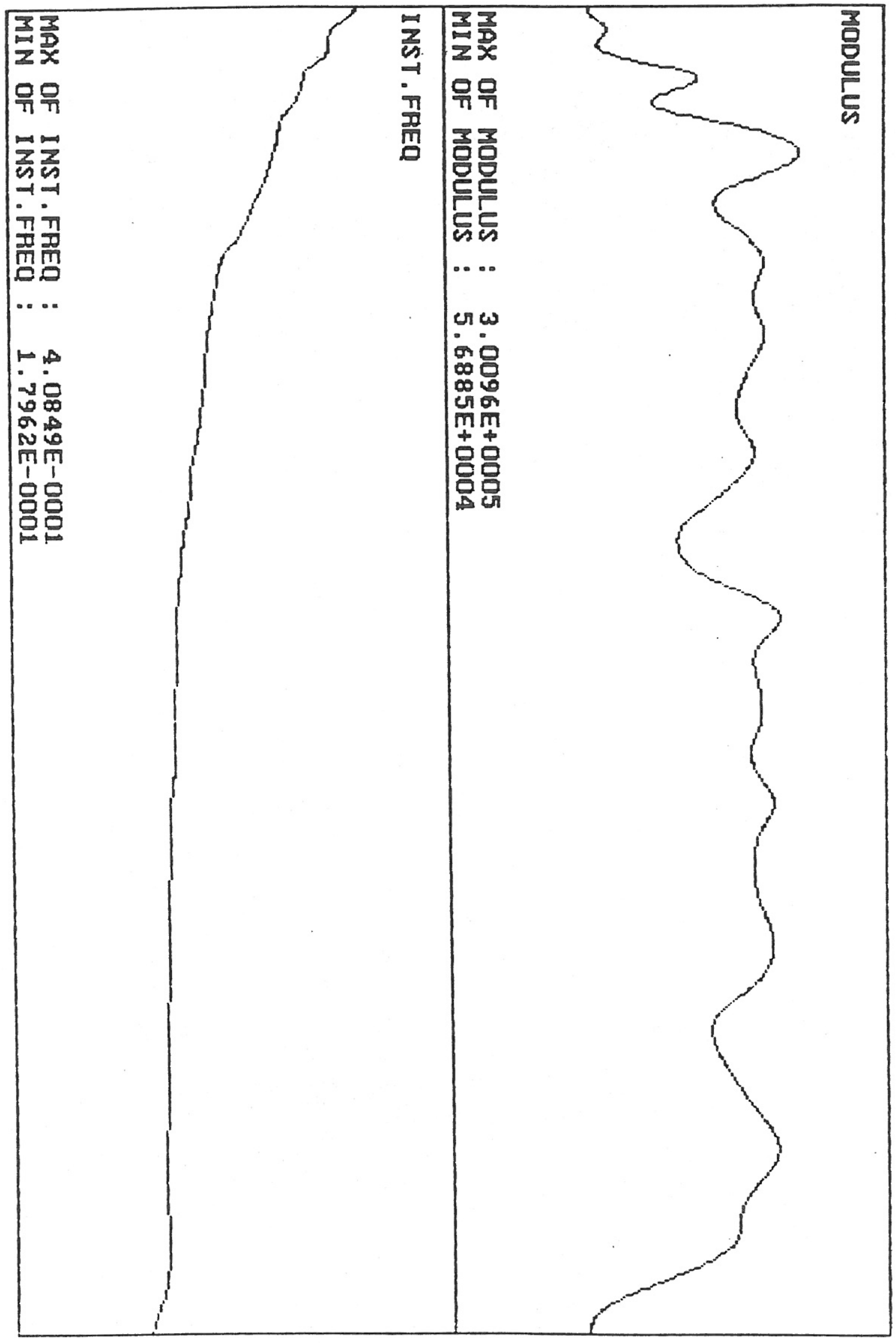

FIG. VI-2-b 


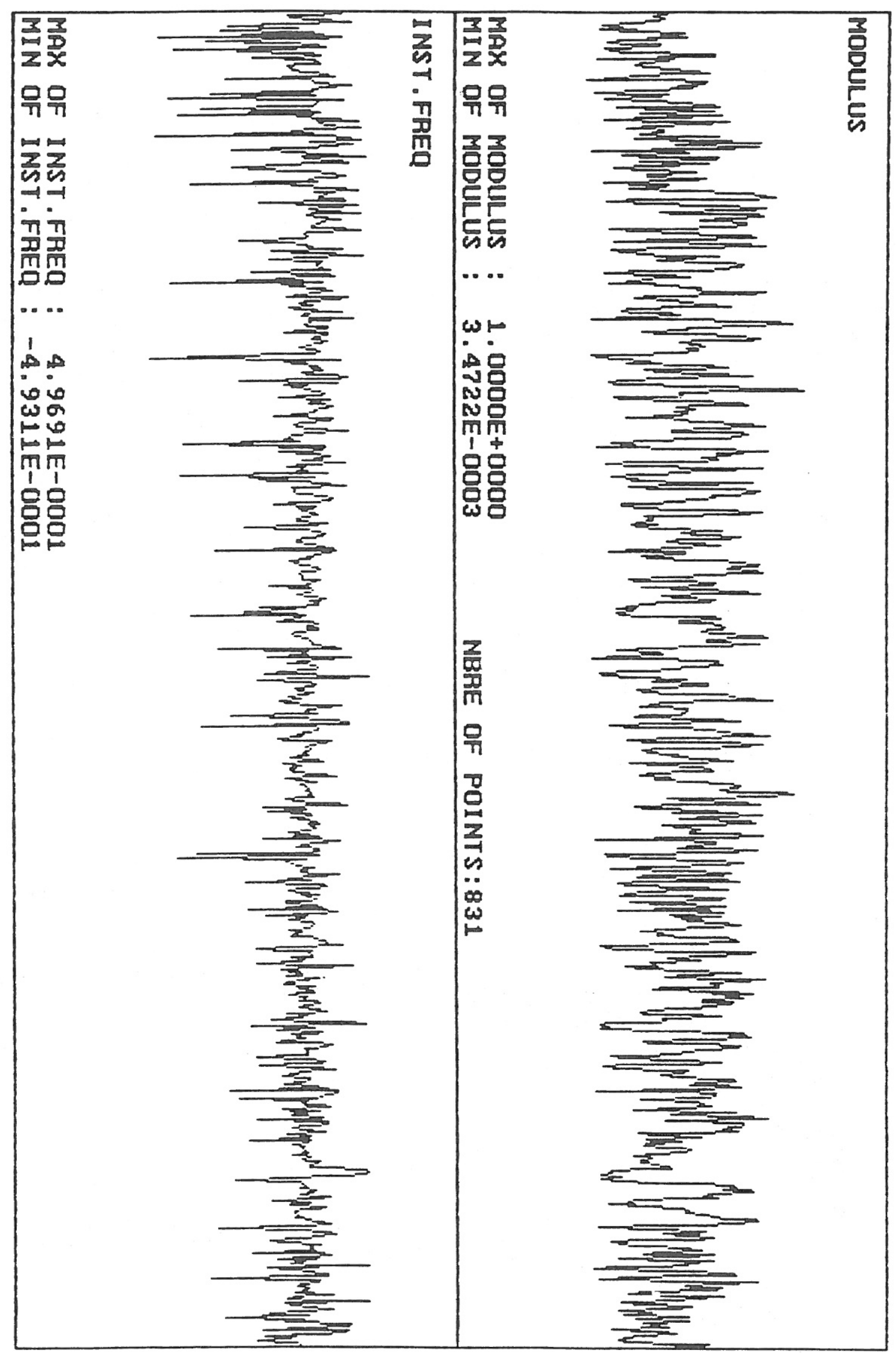

FIG. VI-3 


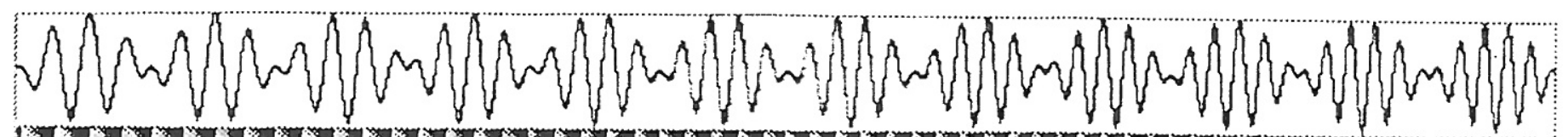
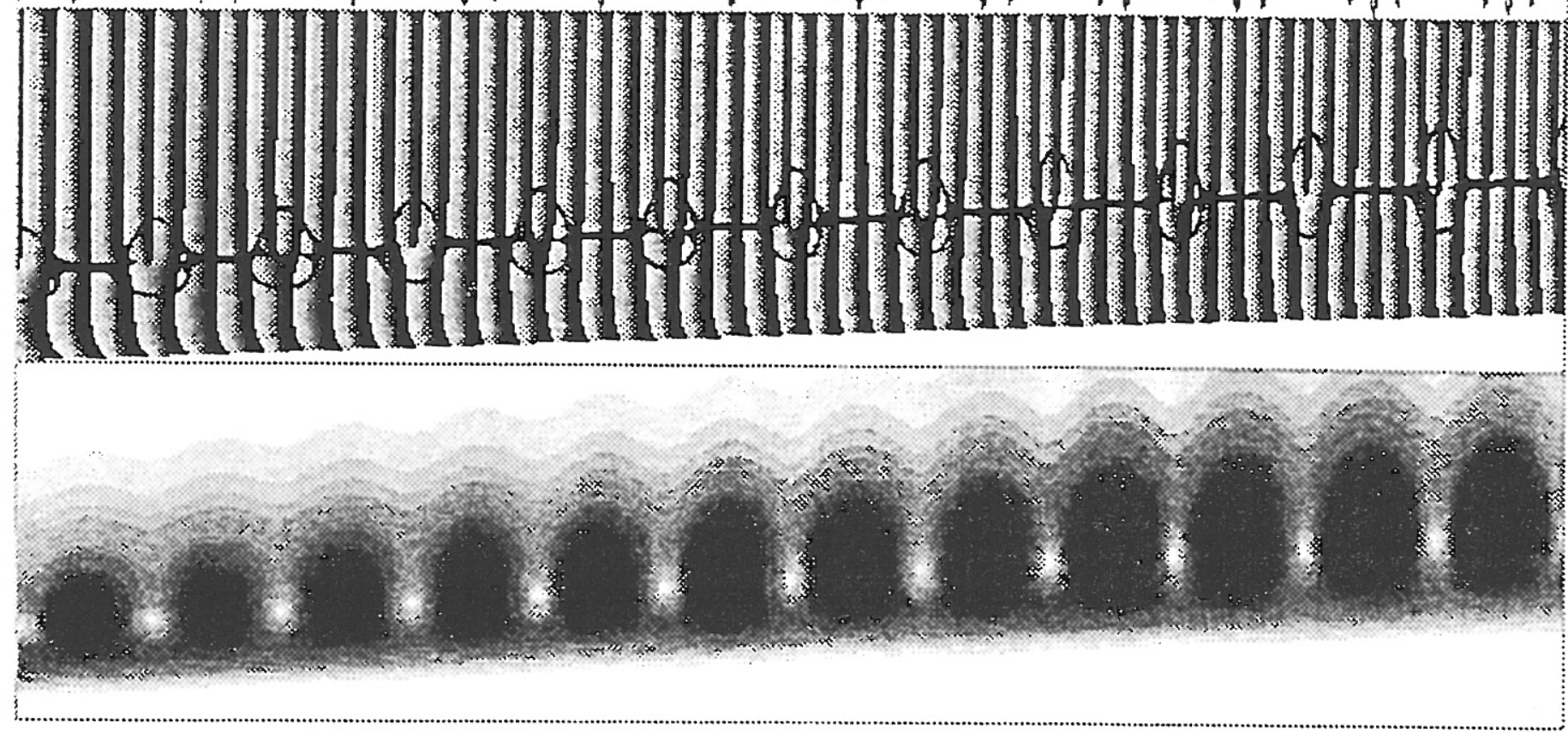


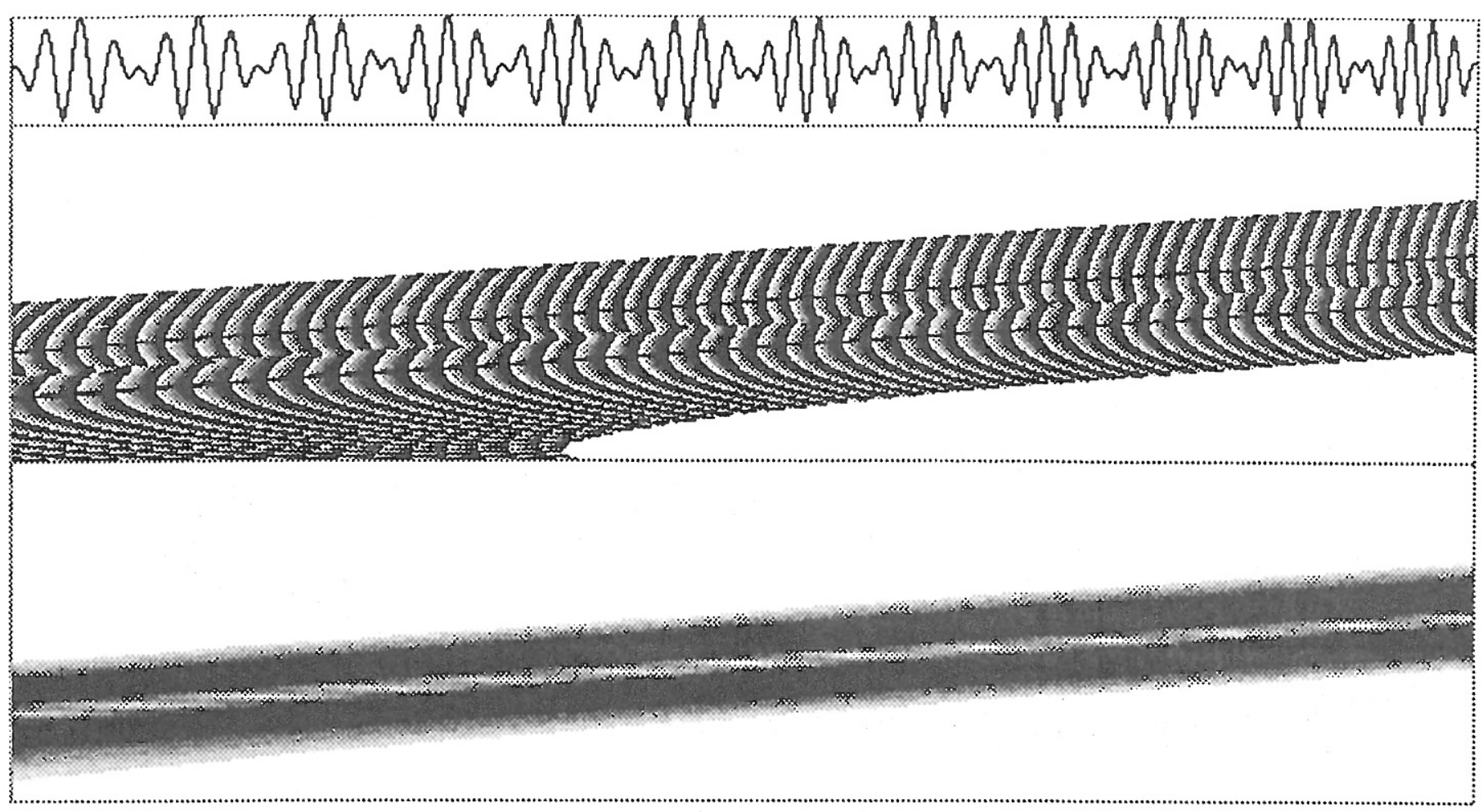

FIG. VI-5 


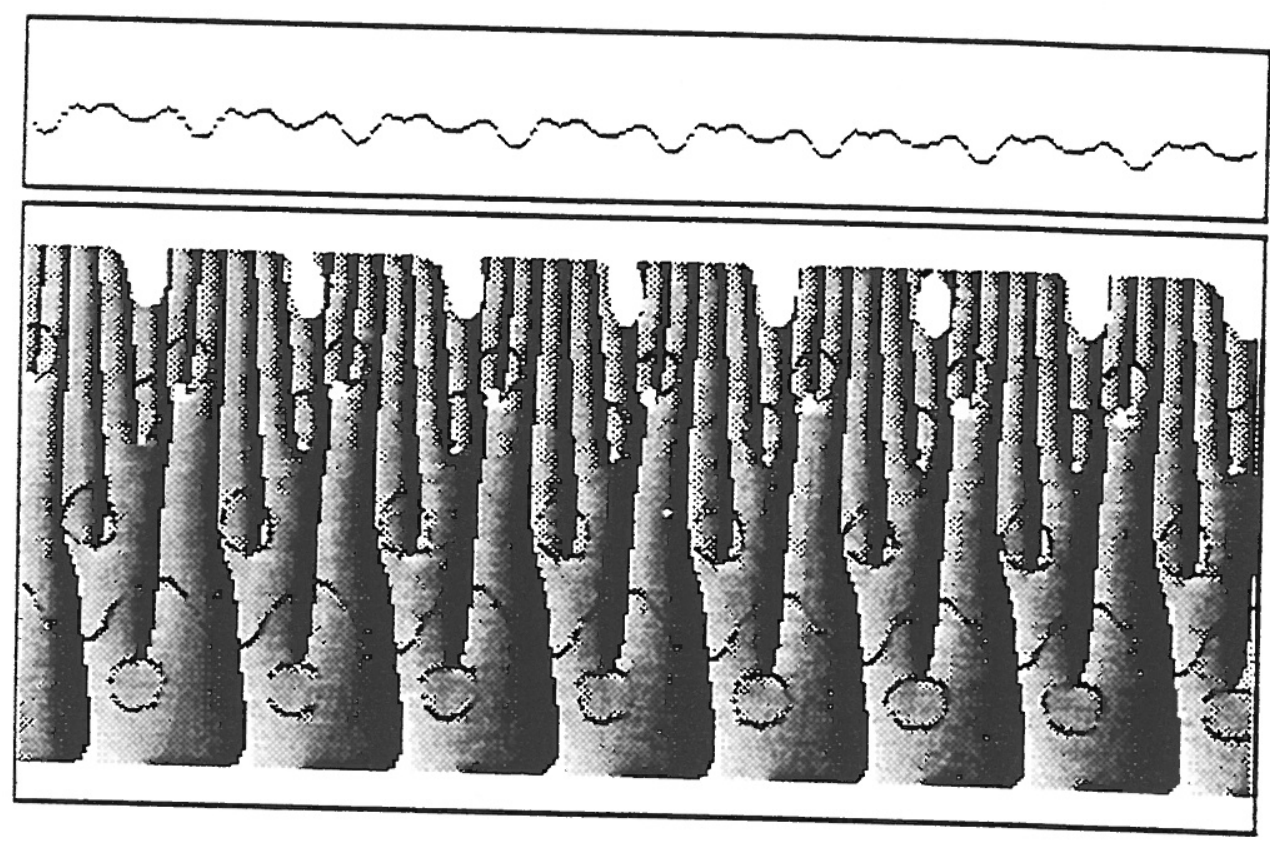

FIG. VI-6-a 

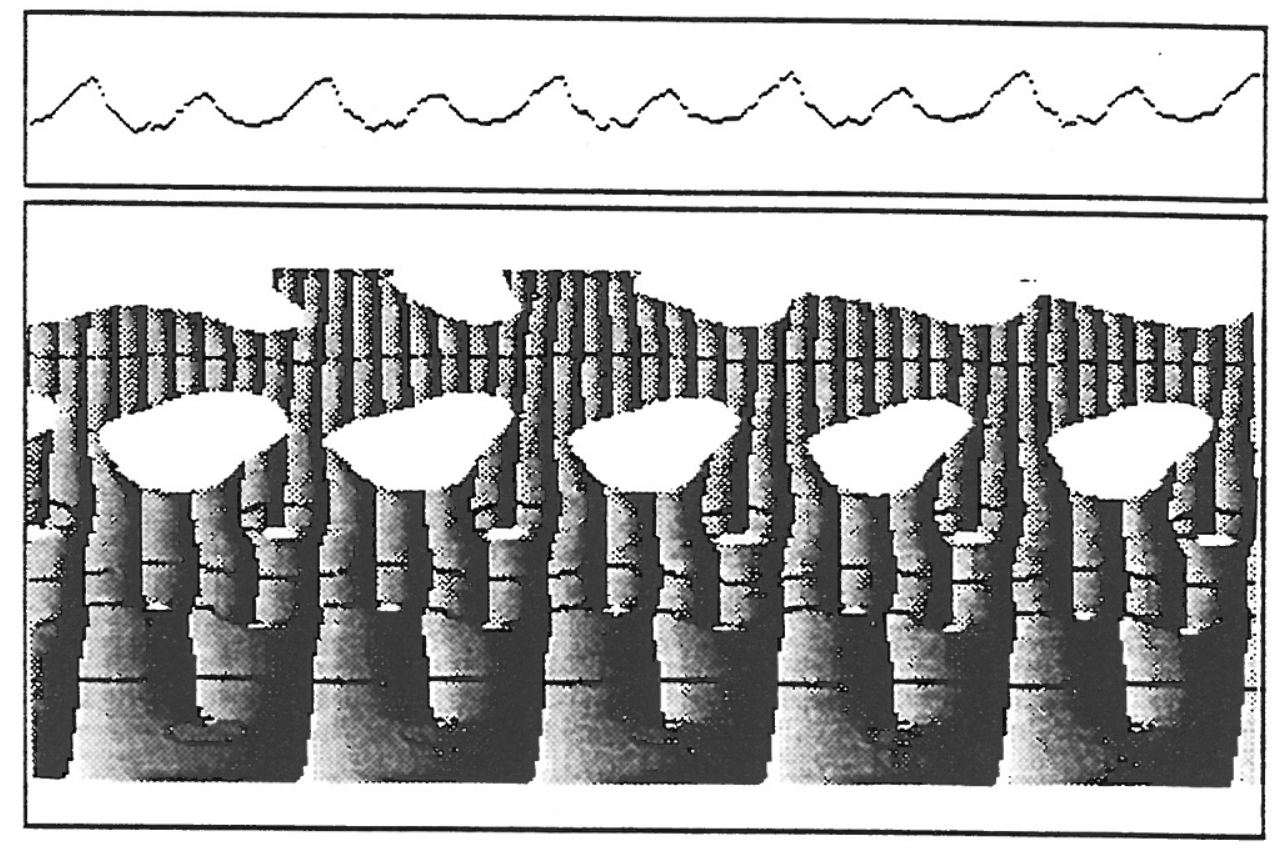

FIG. VI-6-b 


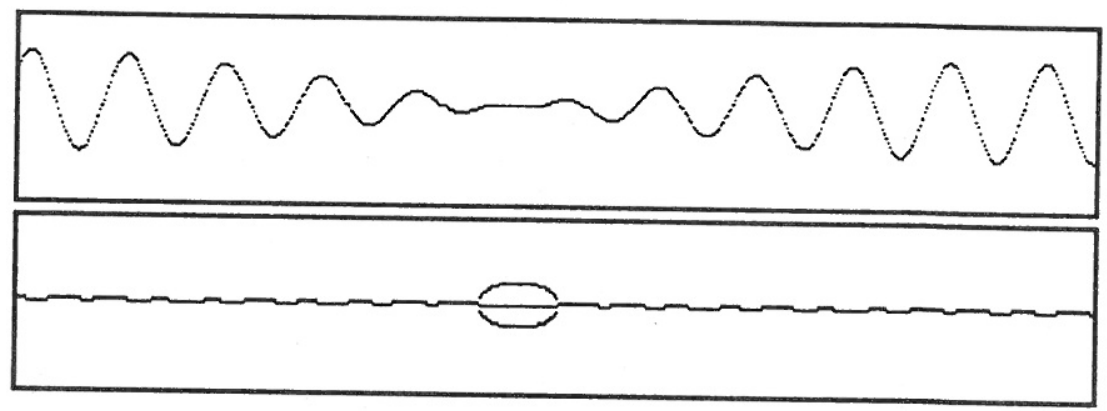

FIG. VI-7
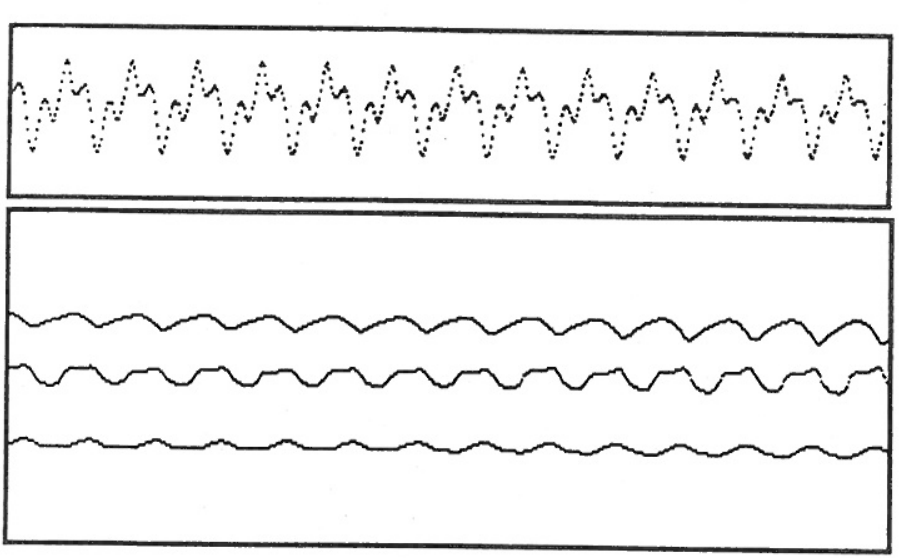

FIG. VI-8-a

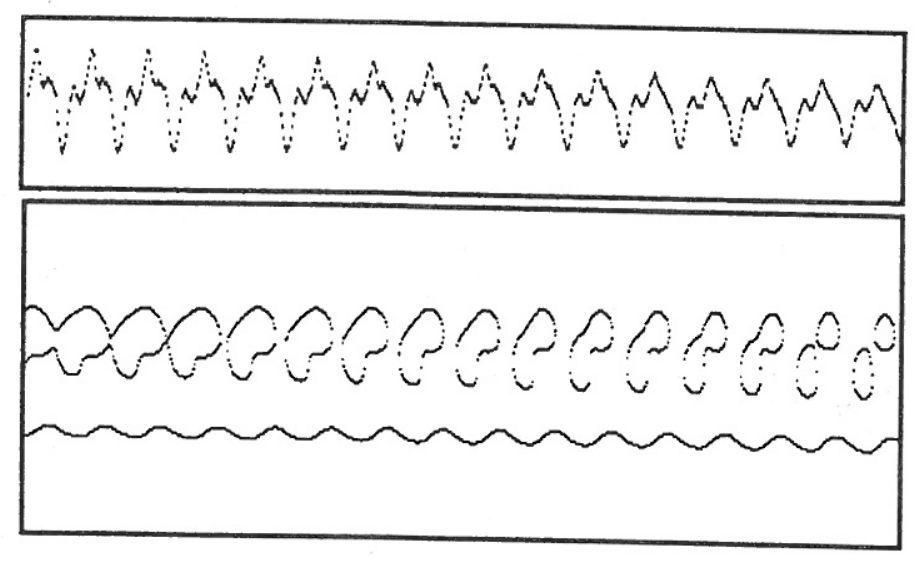

F.IG. VI-8-b 


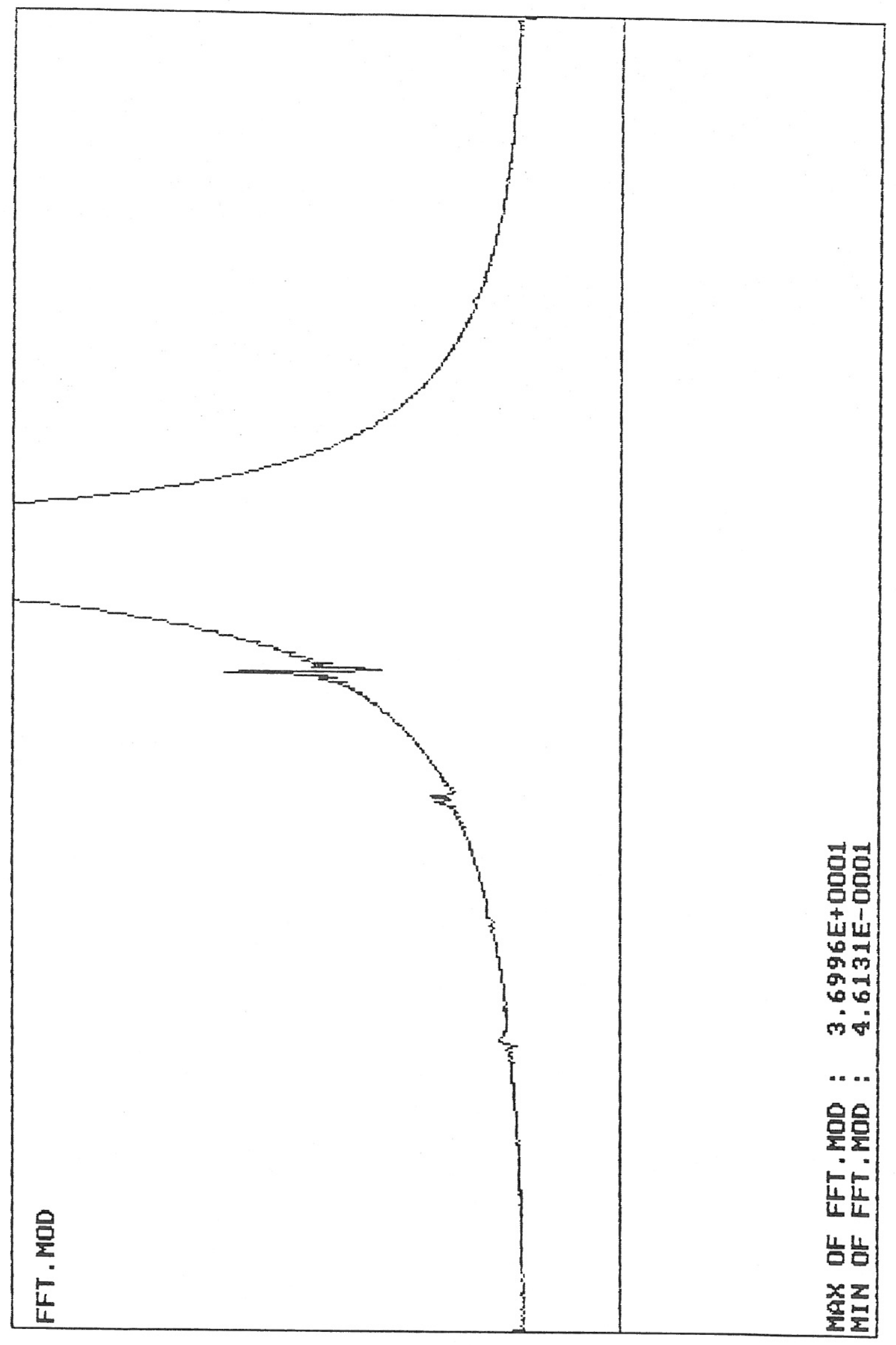

FIG. VI-9-a 


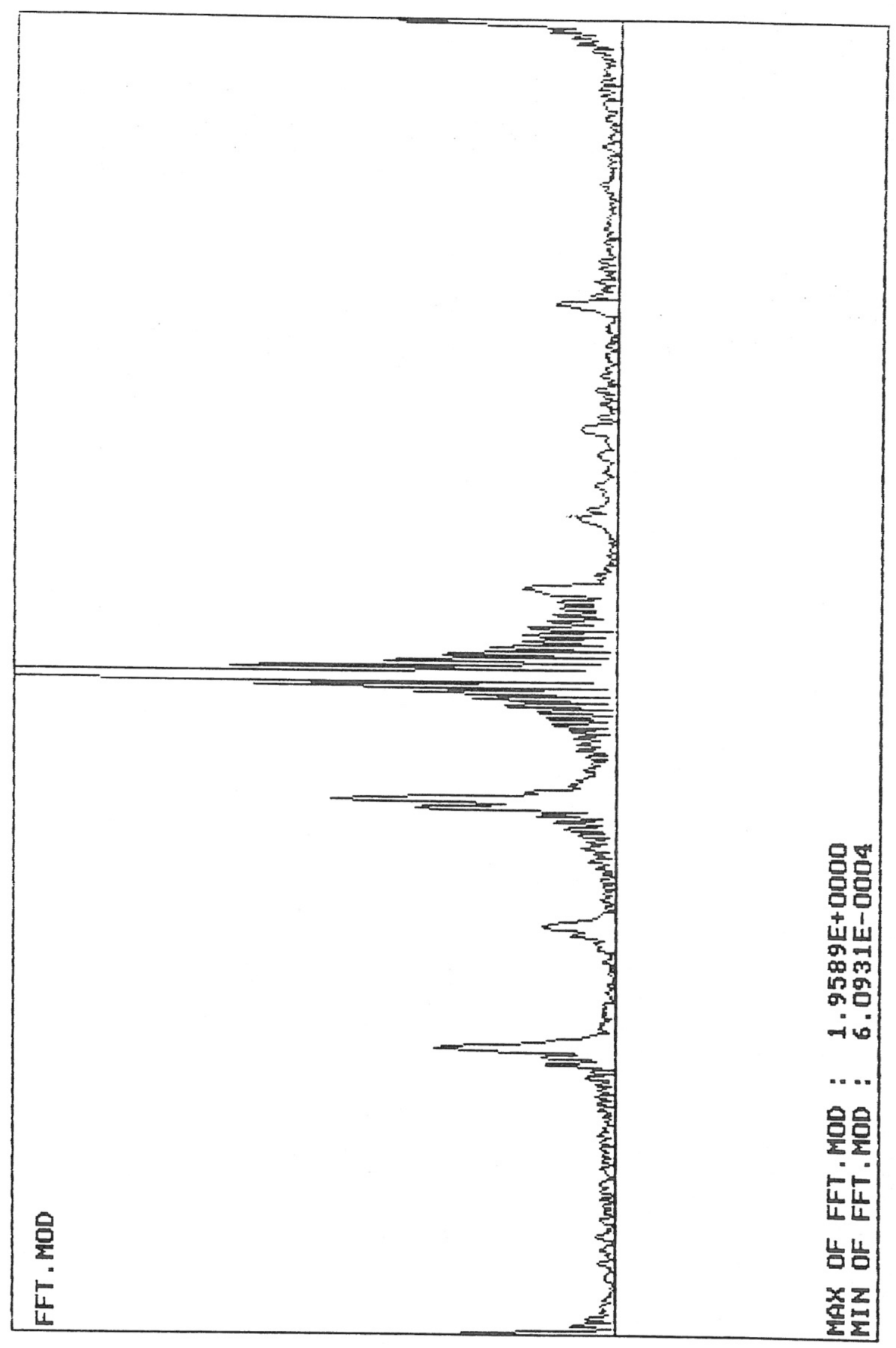

FIG. VI-9-b 


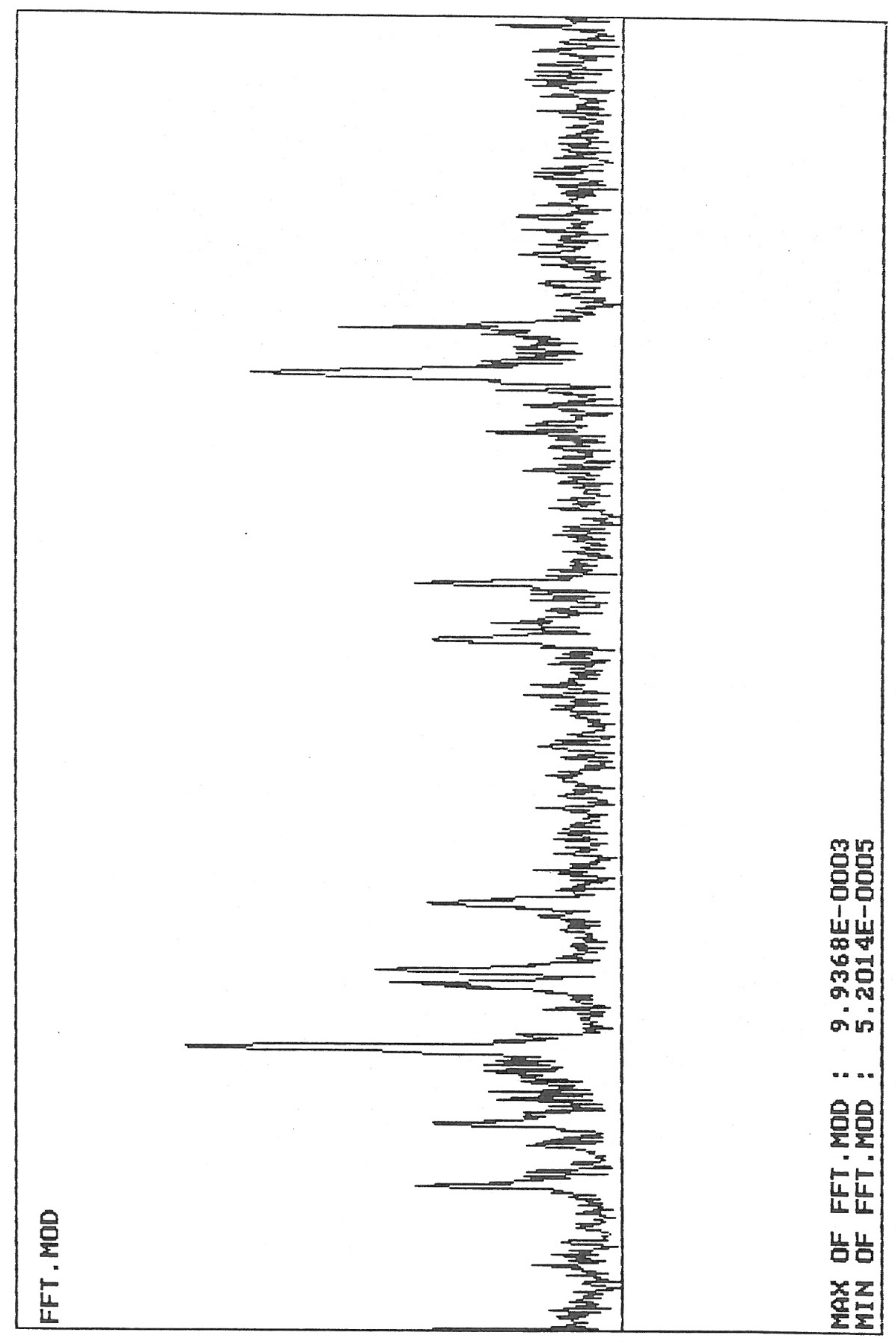

FIG. VI-10-a 


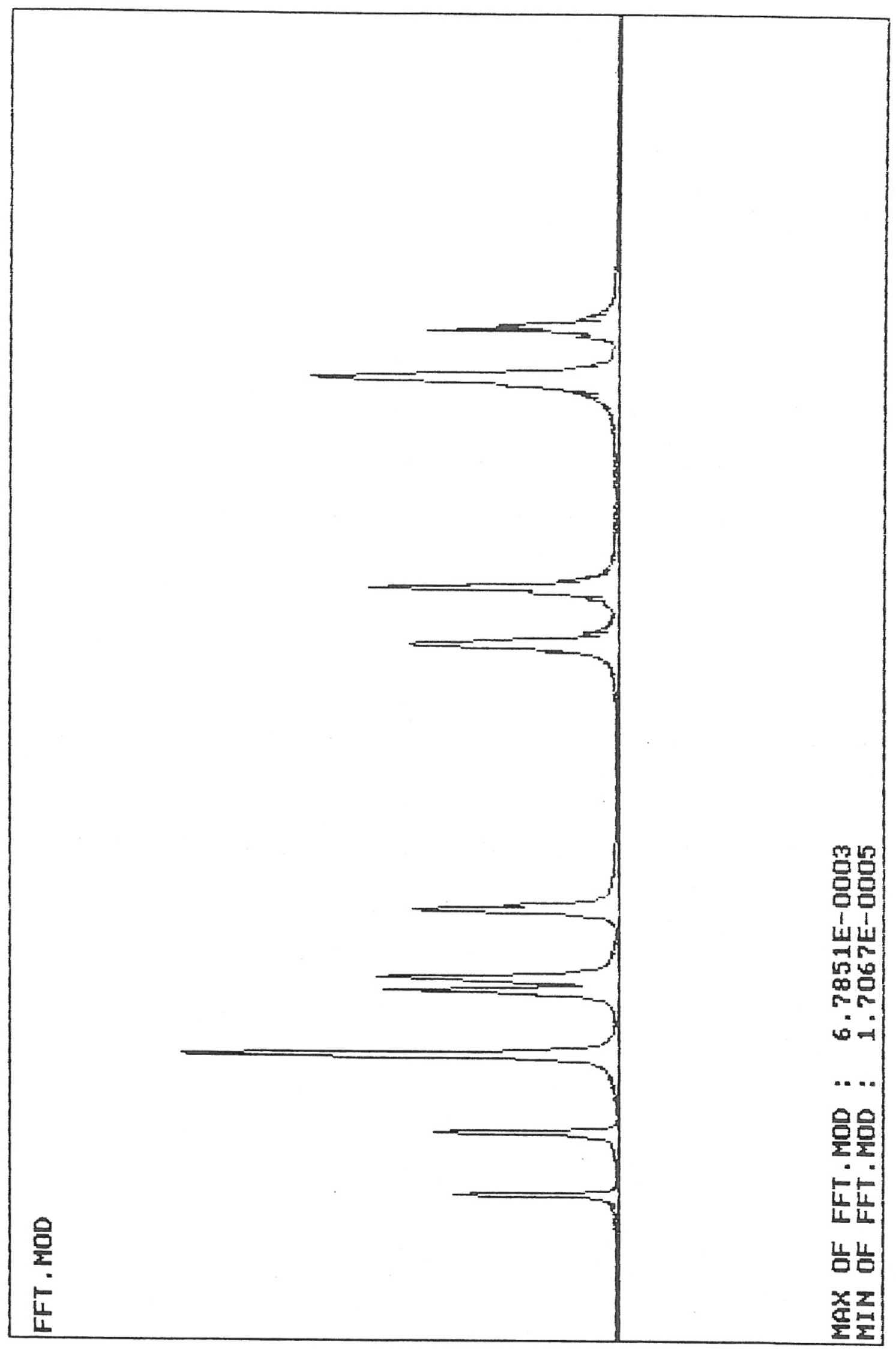

FIG. VI-10-b 


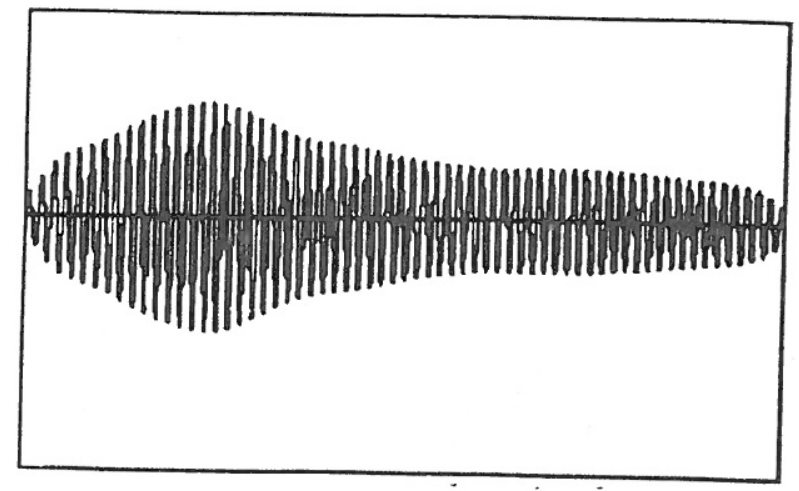

FIG. VI-11-a

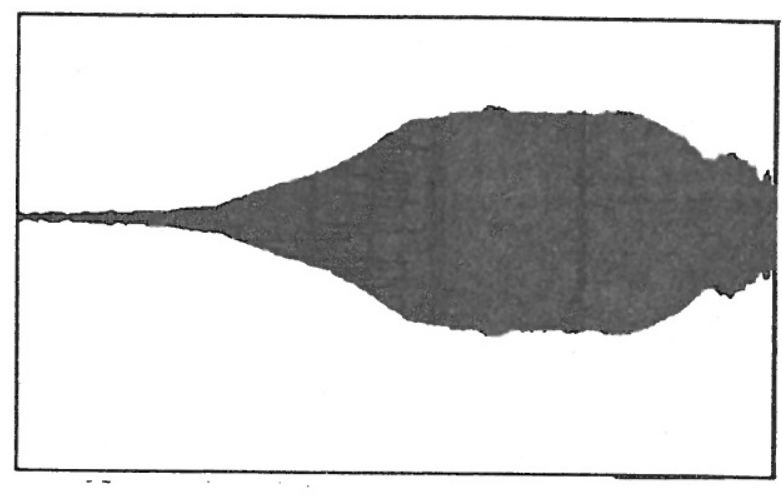

FIG. VI-11-b

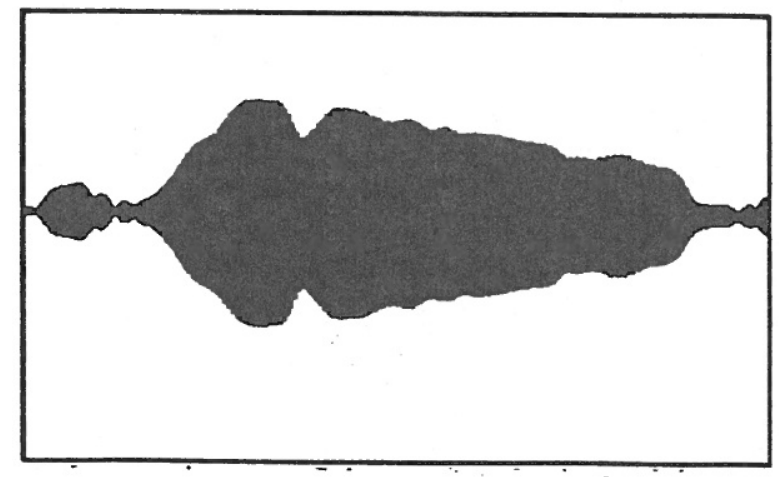

FIG. VI-11-c 


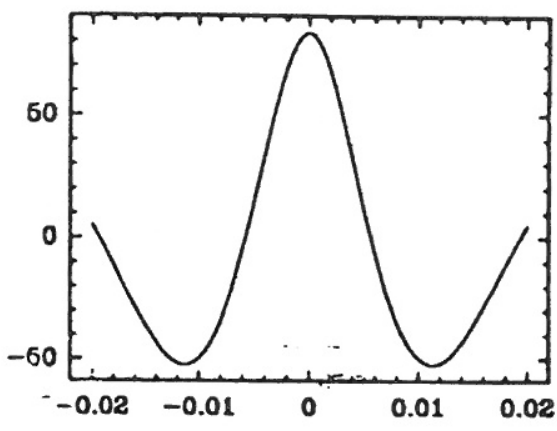

FIG. VII-1-a

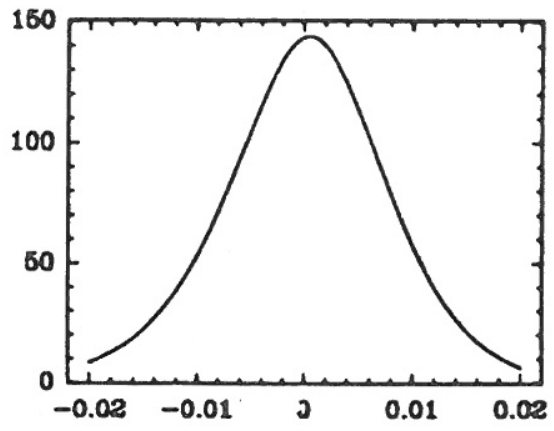

FIG. VII-1-b

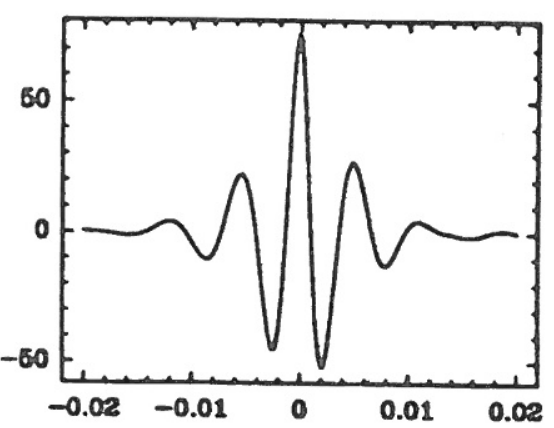

FIG. VII-1-c

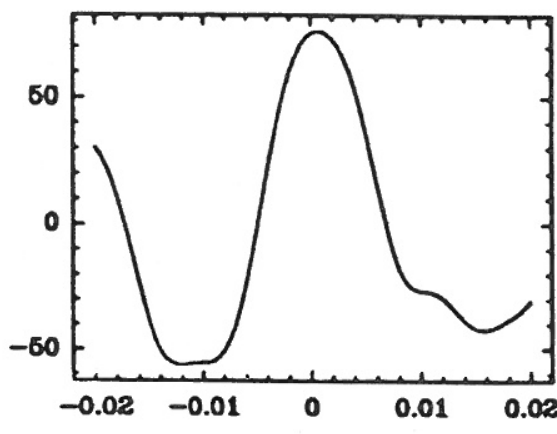

FIG. VII-2-a

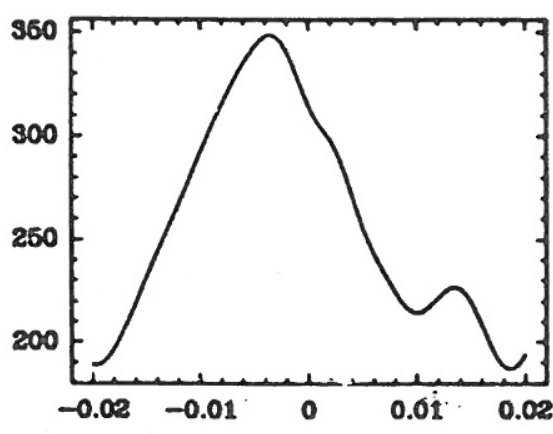

FIG. VII-2-b

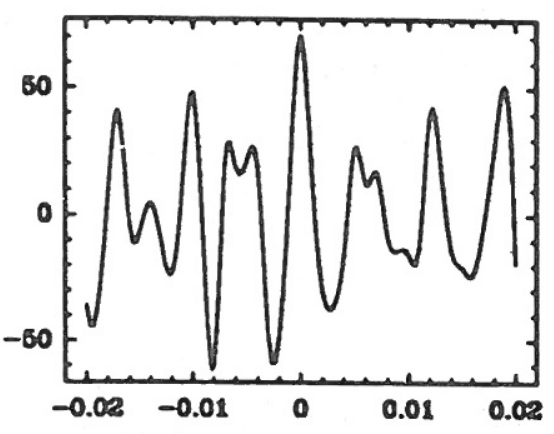

FIG. VII-2-C 


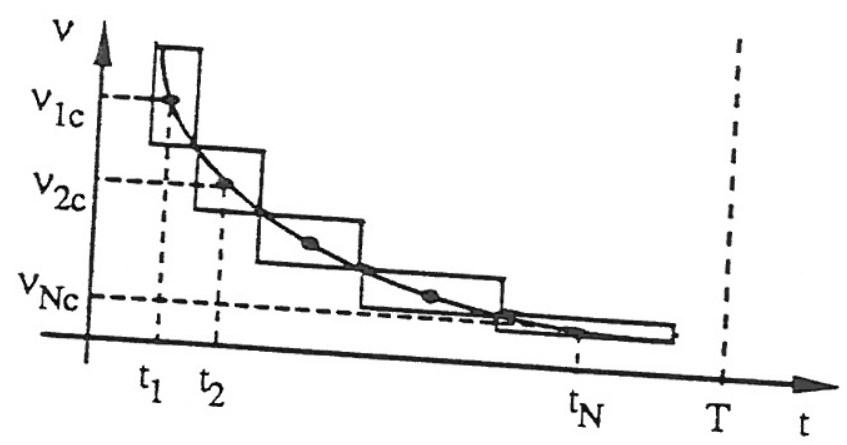

FIG. VII-3-a 


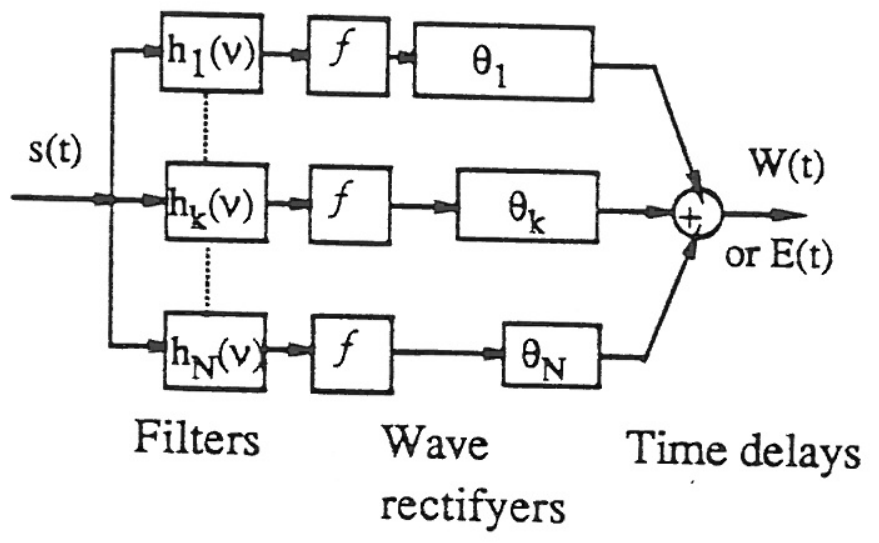

FIG. VII-3-b 


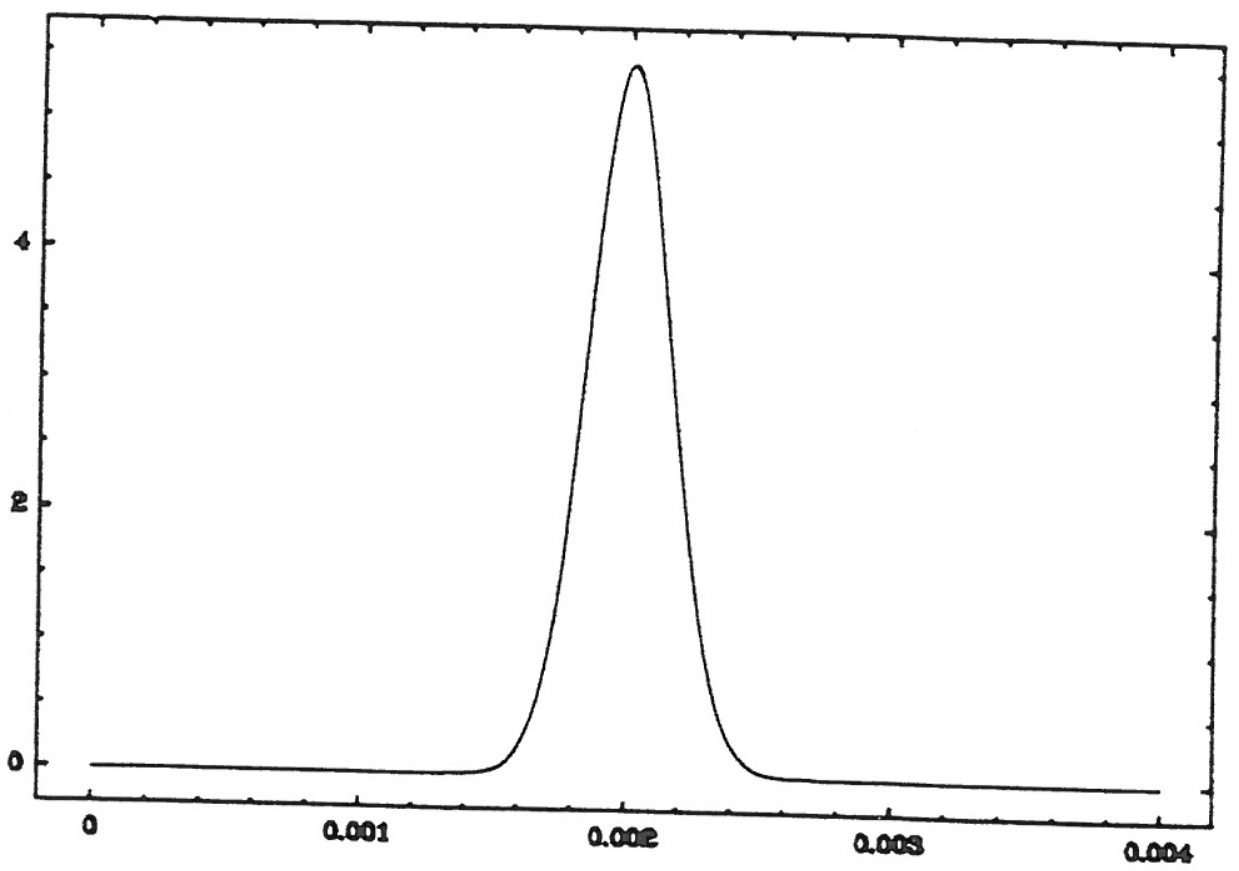

FIG. VII-4-a 


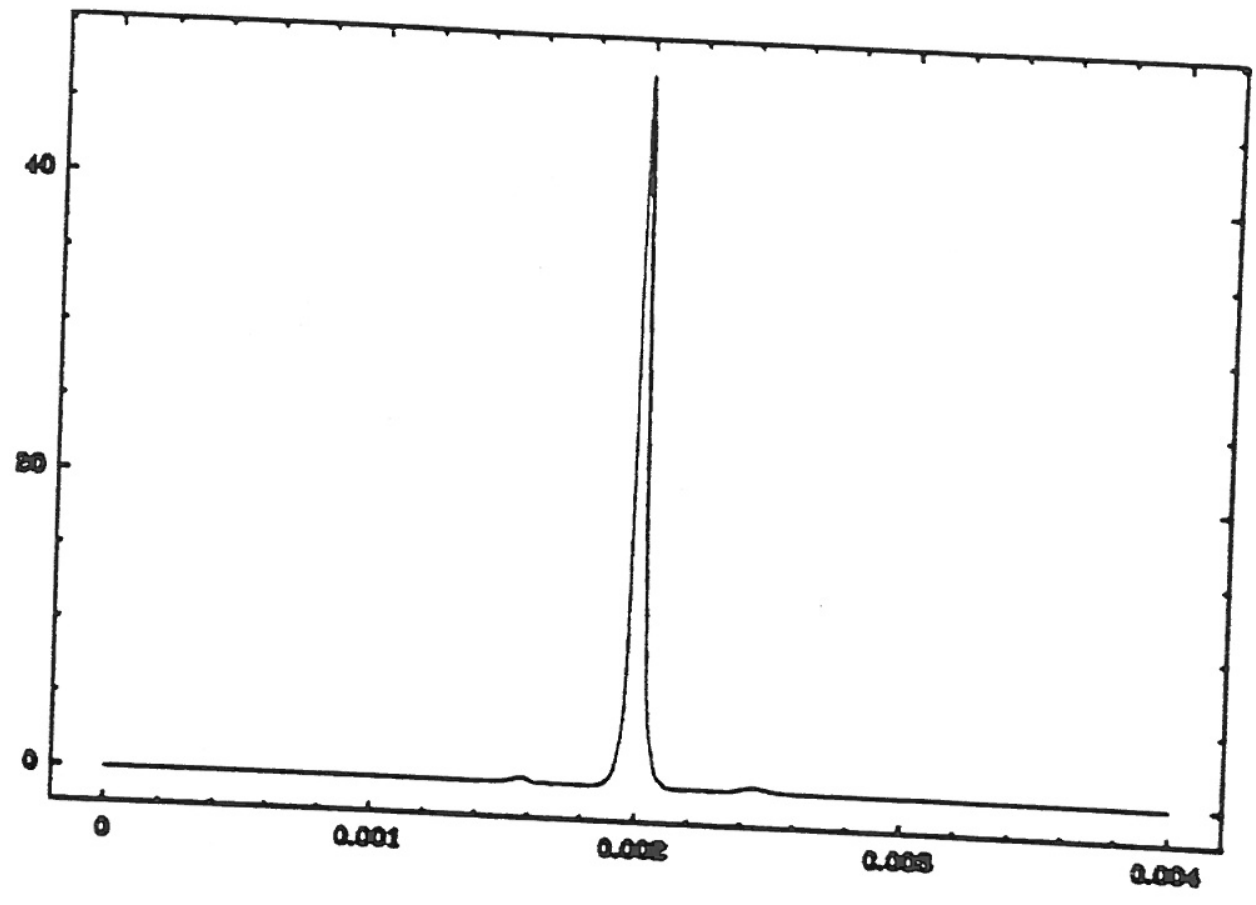

FIG. VII-4-b 


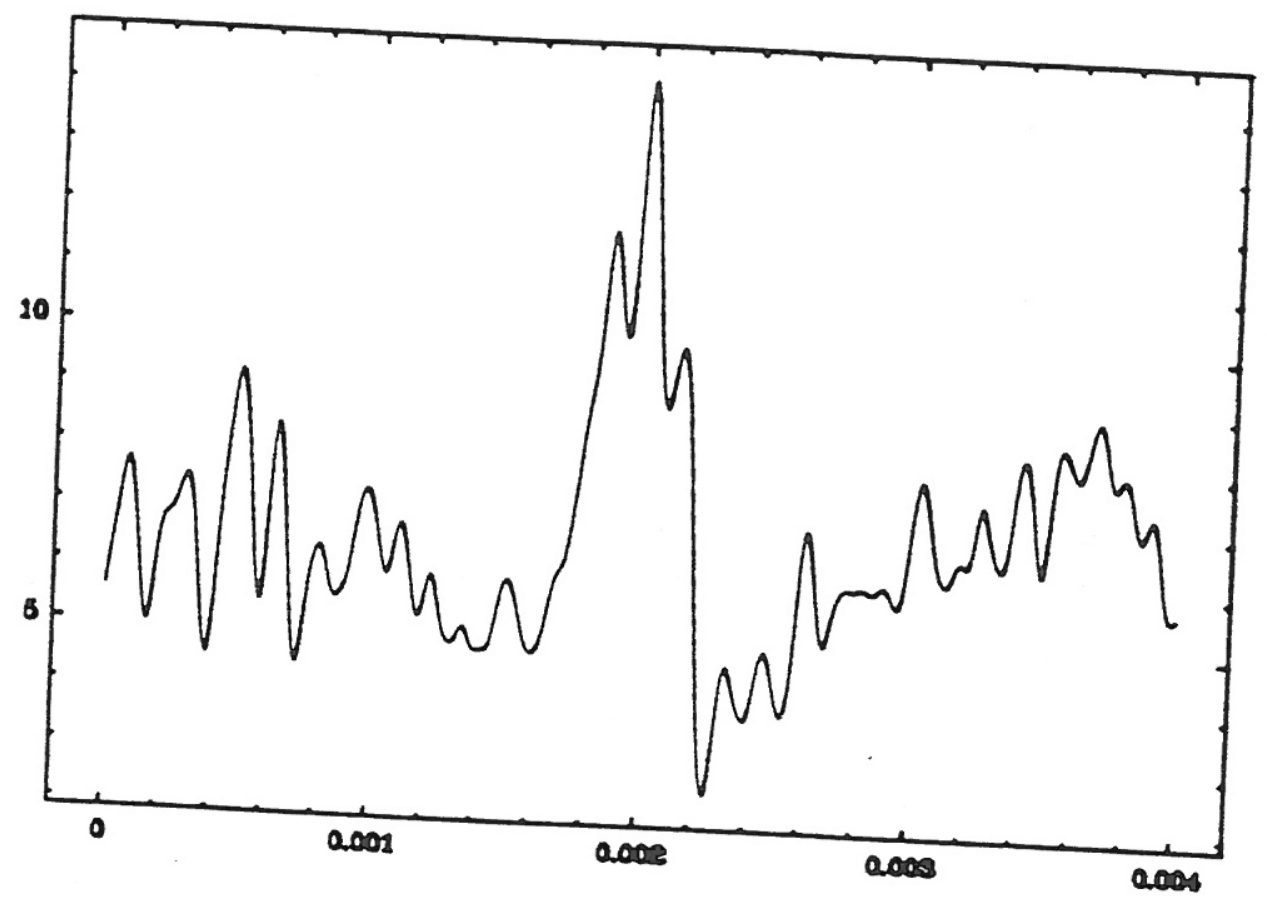

FIG. VII-5-a 


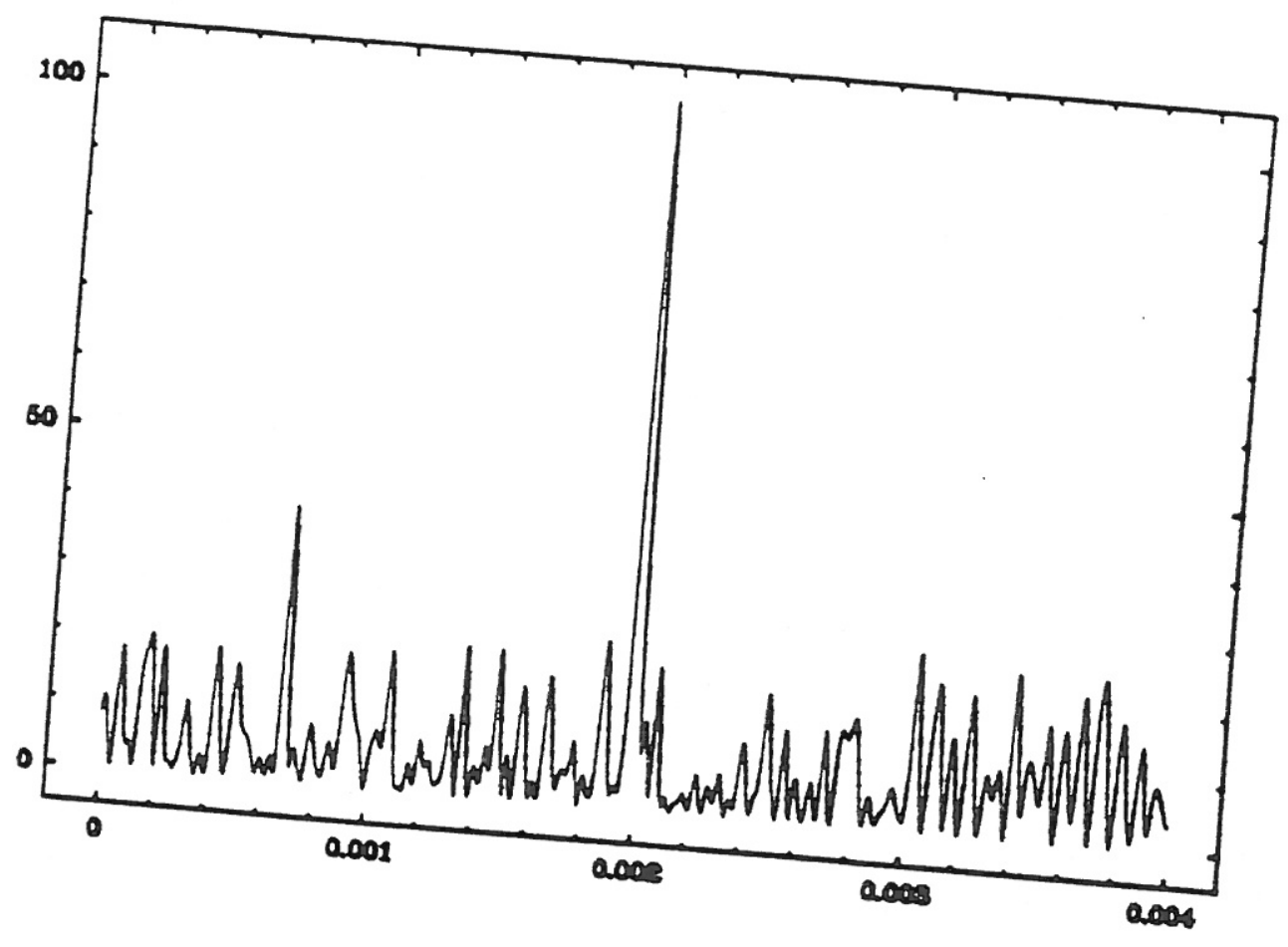

FIG. VII-5-b 